\title{
Non-renormalized solutions to the continuity equation
}

\author{
Stefano Modena ${ }^{1}$. László Székelyhidi Jr. ${ }^{1}$
}

Received: 16 August 2018 / Accepted: 30 September 2019 / Published online: 9 November 2019

(c) The Author(s) 2019

\section{Abstract}

We show that there are continuous, $W^{1, p}(p<d-1)$, incompressible vector fields for which uniqueness of solutions to the continuity equation fails.

Mathematics Subject Classification Primary 35A02 - Secondary 35F10

\section{Introduction}

In this paper we consider the continuity equation

$$
\begin{aligned}
\partial_{t} \rho+\operatorname{div}(\rho u) & =0, \\
\operatorname{div} u & =0,
\end{aligned}
$$

in a $d$-dimensional periodic domain, $d \geq 3$, for a time-dependent incompressible vector field $u:[0,1] \times \mathbb{T}^{d} \rightarrow \mathbb{R}^{d}$ and an unknown density $\rho:[0,1] \times \mathbb{T}^{d} \rightarrow \mathbb{R}$. Here and in the sequel $\mathbb{T}^{d}=\mathbb{R}^{d} / \mathbb{Z}^{d}$ is the $d$-dimensional flat torus. We will also always assume, without loss of generality, that the time interval is $[0,1]$. We prove in these notes the following theorem.

Theorem 1.1 (Non-uniqueness for Sobolev and continuous vector fields). Let $\varepsilon>0, \bar{\rho} \in$ $C^{\infty}\left(\mathbb{T}^{d}\right)$ with $\int_{\mathbb{T}^{d}} \bar{\rho} d x=0$. Then there exist

$$
\rho \in C\left([0,1] ; L^{1}\left(\mathbb{T}^{d}\right)\right), \quad u \in C\left([0,1] \times \mathbb{T}^{d}\right) \cap \bigcap_{1 \leq p<d-1} C\left([0,1] ; W^{1, p}\left(\mathbb{T}^{d}\right)\right)
$$

such that $(\rho, u)$ is a weak solution to $(1)$ and $\rho(0) \equiv 0$ at $t=0, \rho(1) \equiv \bar{\rho}$ at $t=1$ and

$$
\max _{(t, x) \in[0,1] \times \mathbb{T}^{d}}|u(t, x)| \leq \varepsilon .
$$

Communicated by L. Ambrosio.

\section{László Székelyhidi Jr.}

laszlo.szekelyhidi@math.uni-leipzig.de

Stefano Modena

stefano.modena@math.uni-leipzig.de

1 Institut für Mathematik, Universität Leipzig, 04109 Leipzig, Germany 
By weak solution we mean solution in the sense of distributions.

We have chosen the periodic setting for simplicity and to emphasize that the key phenomenon we are studying is the effect of (local) low regularity. As with other applications of convex integration (e.g. $[9,14]$ ), we expect that analogous statements hold also in the full space $\mathbb{R}^{d}$.

It is well known that the theory of classical solutions to (1) is closely connected to the ordinary differential equation

$$
\begin{aligned}
\partial_{t} X(t, x) & =u(t, X(t, x)), \\
X(0, x) & =x,
\end{aligned}
$$

via the formula $\rho(t) \mathcal{L}^{d}=X(t)_{\sharp}\left(\rho(0) \mathcal{L}^{d}\right)$ or, equivalently, due to the incompressibility,

$$
\rho(t, X(t, x))=\rho(0, x) .
$$

In particular, for Lipschitz vector fields $u$ the well-posedness theory for (1) follows from the Cauchy-Lipschitz theory for ordinary differential equations applied to (3).

It is in general of great interest to investigate the existence and uniqueness of weak solutions to the Cauchy problem for (1) in the case of non-smooth vector fields, and the connection to the Lagrangian problem (3), (4). The general question can be formulated as follows. Fix an exponent $r \in[1, \infty]$, denote by $r^{\prime}$ its dual Hölder, $1 / r+1 / r^{\prime}=1$, and assume that a vector field

$$
u \in L^{1}\left(0,1 ; L^{r}\left(\mathbb{T}^{d}\right)\right)
$$

is given. What can be said about existence and uniqueness of weak solutions in the class of densities

$$
\rho \in L^{\infty}\left(0,1 ; L^{r^{\prime}}\left(\mathbb{T}^{d}\right)\right) ?
$$

The choice of class (6) for $\rho$ is motivated by the fact that for classical solutions to (1) every spatial $L^{r^{\prime}}$ norm is preserved in time. Once (6) is fixed, the choice of the class (5) for $u$ is natural, since in this way $\rho u \in L^{1}\left((0,1) \times \mathbb{T}^{d}\right)$ and thus the notion of distributional solution to (1) is well defined.

While existence of weak solutions can be easily shown under the assumptions (5), (6), the uniqueness question is much harder. In 1989 DiPerna and Lions [11] proved that uniqueness holds in the class (6) if

$$
D u \in L^{1}\left(0,1 ; L^{r}\left(\mathbb{T}^{d}\right)\right)
$$

i.e. if $u$ enjoys Sobolev regularity with exponent $r$. Moreover, in this case, the incompressibility assumption can be relaxed to $\operatorname{div} u \in L^{\infty}$. In the class of bounded densities the uniqueness result was later extended by Ambrosio [1] in 2004, for fields $u \in L^{1}(0,1 ; B V)$ with div $u \in L^{\infty}$ and very recently by Bianchini and Bonicatto [3] in 2017 in the case of $B V$ nearly incompressible vector fields.

In all of these results an important additional feature is the connection to a suitable extension to (3), i.e. the link between the Eulerian and the Lagrangian picture. More precisely, under assumption (7), there exists a unique distributional solution to (3) for a.e. $x$, such that $x \mapsto X(t, x)$ is measure preserving for all $t$ (assuming div $u=0$ ): such flow map is called regular Lagrangian flow (see [2] for a general discussion). Then the unique solution to the continuity or the transport equation is given by (4), as in the smooth case.

On the other side, several non-uniqueness counterexamples are known, but they mainly concern the case when the field is "very far" from being incompressible (e.g. $\operatorname{div} u \notin L^{\infty}$, 
see [11]) or the case when no bounds on one full derivative of $u$ are available (see, for instance, the counterexample in [11] for a field $u \in L^{1}\left(0,1 ; W^{s, 1}\right)$ for every $s<1$, but $u \notin L^{1}\left(0,1 ; W^{1,1}\right)$ or the counterexample in [10] for a field $u \in L^{1}(\varepsilon, 1 ; B V)$ for every $\varepsilon>0$, but $\left.u \notin L^{1}(0,1 ; B V)\right)$. In all these counterexamples, however, non-uniqueness for the PDE (1) is a consequence of a Lagrangian non-uniqueness for the associated ODE (3). We refer to [16] and to [2] for a more detailed discussion.

Very recently, we proved in [16] the analog of Theorem 1.1, for fields and densities in the class

$$
\rho \in C\left([0,1] ; L^{r^{\prime}}\left(\mathbb{T}^{d}\right)\right), \quad u \in C\left([0,1] ; L^{r}\left(\mathbb{T}^{d}\right)\right) \cap C\left([0,1] ; W^{1, p}\left(\mathbb{T}^{d}\right)\right),
$$

with

$$
r \in(1, \infty), \quad p \in[1, \infty)
$$

and

$$
\frac{1}{r^{\prime}}+\frac{1}{p}>1+\frac{1}{d-1} .
$$

The result in [16] shows that uniqueness can fail even for incompressible, Sobolev vector fields (i.e. fields for which the Lagrangian problem (3) is well posed, in the sense of the regular Lagrangian flow), if the integrability exponent $p$ of $D u$ is much lower than the one provided in (7) by DiPerna and Lions' theory, as specified in (9).

The end-point $r=\infty$, corresponding in (9) to $p<d-1$, is excluded in [16]. The main result of this notes, namely Theorem 1.1, shows that such end-point case can indeed be reached and, in addition, quite surprisingly, the vector field produced by Theorem 1.1 is continuous in time and space, not only bounded.

We postpone to Sect. 2 a technical discussion about why the case $r=\infty$ was out of reach in [16] and which new ideas are introduced in these notes to deal with such problem.

We would like now to briefly comment about the continuity of the vector field $u$ produced by Theorem 1.1. It was observed by Caravenna and Crippa [7] that the boundedness or the continuity of the vector field (in addition to some Sobolev regularity) could play a key role in the uniqueness problem in the class of integrable densities $\rho \in L^{1}\left((0,1) \times \mathbb{T}^{d}\right)$. It thus turns out to be a very interesting question to ask if, in fact, boundedness or continuity plus Sobolev regularity are enough to guarantee uniqueness. Theorem 1.1 shows that this is not the case, if the integrability of $D u$ is lower than a dimensional threshold (precisely, $d-1){ }^{1}$

The idea that the boundedness or the continuity of $u$ can play a crucial role in the uniqueness problem is confirmed by the fact that the majority of the result concerning existence and uniqueness of the regular Lagrangian flow associated to a Sobolev or $B V$ vector field $u$ assume that $u \in L^{\infty}$ (see, for instance, the recent survey [2]).

On a different point of view, it is a classical result (see, for instance, [12]) that the boundedness of $u$, even without any further Sobolev regularity, is enough to have uniqueness, if a small viscosity is added to the continuity equation:

$$
\partial_{t} \rho+\operatorname{div}(\rho u)=v \Delta \rho, \quad v>0,
$$

while in [16] we showed that uniqueness for (10) can drastically fail is $u$ is Sobolev, but not bounded.

1 While the present paper was under review, G. Sattig and the first author, relying on the ideas introduced in the present paper, showed in [15] that this dimensional threshold can be raised up to $d$. 
The result in Theorem 1.1 is quite surprising, even in comparison with our previous result in [16]. Indeed, for vector fields produced by Theorem 1.1 the Lagrangian picture is very well behaved: first, the Sobolev regularity implies the existence and uniqueness of the regular Lagrangian flow. Second, the continuity of the field implies that the trajectories provided by the regular Lagrangian flow are $C^{1}$ in time (and this was not the case for the fields produced in [16]). Third, the bound (2) means that the length of each trajectory is at most $\varepsilon>0$, i.e. particles almost don't move (and, again, this was not the case for the fields produced in [16]). Observe also that $\varepsilon$ in (2) depends neither on the length of the time interval $[0,1]$ nor on the $L^{1}$ distance between the initial and the final datum $\|\rho(1)-\rho(0)\|_{L^{1}\left(\mathbb{T}^{d}\right)}=\|\bar{\rho}\|_{L^{1}\left(\mathbb{T}^{d}\right)}$. Nevertheless uniqueness in the Eulerian world is completely lost.

We conclude this introduction observing that Theorem 1.1 is an immediate application of the following theorem, whose proof is the topic of all next sections.

Theorem 1.2 Let $\varepsilon>0$. Let $\rho_{0}:[0,1] \times \mathbb{T}^{d} \rightarrow \mathbb{R}, u_{0}:[0,1] \times \mathbb{T}^{d} \rightarrow \mathbb{R}^{d}$ be smooth with

$$
\begin{aligned}
\int_{\mathbb{T}^{d}} \rho_{0}(0, x) d x & =\int_{\mathbb{T}^{d}} \rho_{0}(t, x) d x, \\
\operatorname{div} u_{0} & =0,
\end{aligned}
$$

for every $t \in[0,1]$. Set

$$
E:=\left\{t \in[0,1]: \partial_{t} \rho_{0}(t)+\operatorname{div}\left(\rho_{0}(t) u_{0}(t)\right)=0\right\} .
$$

Then there exist $\rho:[0,1] \times \mathbb{T}^{d} \rightarrow \mathbb{R}, u:[0,1] \times \mathbb{T}^{d} \rightarrow \mathbb{R}^{d}$ such that

(a) $\rho$, $u$ have the following regularity:

$$
\rho \in C\left([0,1] ; L^{1}\left(\mathbb{T}^{d}\right)\right), \quad u \in C\left([0,1] \times \mathbb{T}^{d}\right) \cap \bigcap_{1 \leq p<d-1} C\left([0,1] ; W^{1, p}\left(\mathbb{T}^{d}\right)\right) ;
$$

(b) $(\rho, u)$ is a weak solution to $(1)$;

(c) for every $t \in E, \rho(t)=\rho_{0}(t), u(t)=u_{0}(t)$;

(d) $\rho$ is $\varepsilon$-close to $\rho_{0}$ i.e.

$$
\max _{t \in[0,1]}\left\|\rho(t)-\rho_{0}(t)\right\|_{L^{1}\left(\mathbb{T}^{d}\right)} \leq \varepsilon .
$$

Condition (d) can be substituted by the following:

(d') $u$ is $\varepsilon$-close to $u_{0}$ i.e.

$$
\left\|u-u_{0}\right\|_{C^{0}\left([0,1] \times \mathbb{T}^{d}\right)} \leq \varepsilon .
$$

We emphasize that conditions (d) or (d') amount to approximability in a strong norm. This is at variance with the $h$-principle type approximability in a weak norm, as for instance in $[5,8]$. In particular, it is easy to see that conditions (d) and (d') cannot hold simultaneously; Indeed, such a statement would imply that one can construct a sequence $\left(\rho_{k}, u_{k}\right)$ of weak solutions to (1) such that $\left(\rho_{k}, u_{k}, \rho_{k} u_{k}\right) \rightarrow\left(\rho_{0}, u_{0}, \rho_{0} u_{0}\right)$ in $L^{1}$, so that necessarily $\left(\rho_{0}, u_{0}\right)$ is also a weak solution to (1). See also Remark 4.2.

Proof of Theorem 1.1 assuming Theorem 1.2 Let $\chi:[0,1] \rightarrow \mathbb{R}$ be such that $\chi \equiv 0$ on $[0,1 / 4], \chi \equiv 1$ on $[3 / 4,1]$. Apply Theorem 1.2 with $\rho_{0}(t, x):=\chi(t) \bar{\rho}(x), u_{0}=0$. By (c), $\rho(0) \equiv 0$ at $t=0$ and $\rho(1) \equiv \bar{\rho}$ at $t=1$. Moreover, by (d'), $\|u\|_{C^{0}} \leq \varepsilon$. 


\section{Comments on the proof}

We describe in this section what problems arise when one tries to extend the proof provided in [16] to Theorem 1.1, i.e. to the end-point case $r=\infty$ and which new ideas are introduced to solve such problems.

\subsection{Sketch of the paper [16]}

We first briefly sketch the proof provided in [16] for the analog of Theorem 1.1 under the conditions (8), (9). The proof is based on a convex integration scheme, with both oscillations and concentration playing a key role. More precisely, the density $\rho$ and the field $u$ are defined as limit of a sequence $\left(\rho_{q}\right)_{q},\left(u_{q}\right)_{q}$ of smooth approximate solutions to the continuity equation

$$
\partial_{t} \rho_{q}+\operatorname{div}\left(\rho_{q} u_{q}\right)=-\operatorname{div} R_{q},
$$

where $R_{q}$ is a smooth vector field converging strongly to zero

$$
\left\|R_{q}\right\|_{C_{t} L_{x}^{1}} \lesssim \delta_{q}
$$

with $\delta_{q}=2^{-q}$ and $\left(\rho_{q}\right)_{q},\left(u_{q}\right)_{q}$ satisfy

$$
\begin{aligned}
& \sum_{q}\left\|\rho_{q}-\rho_{q-1}\right\|_{C_{t} L_{x}^{r^{\prime}}}<\infty, \\
& \sum_{q}\left\|u_{q}-u_{q-1}\right\|_{C_{t} L_{x}^{r}}<\infty,
\end{aligned}
$$

and

$$
\sum_{q}\left\|D u_{q}-D u_{q-1}\right\|_{C_{t} L_{x}^{p}}<\infty .
$$

In this way $\rho, u$ are a weak solution to (1) and, moreover, they have the desired regularity.

The sequence $\left(\rho_{q}, u_{q}, R_{q}\right)$ is constructed recursively. Assuming $\left(\rho_{q-1}, u_{q-1}, R_{q-1}\right)$ are given, one defines

$$
\rho_{q}=\rho_{q-1}+\vartheta_{q}, \quad u_{q}=u_{q-1}+w_{q}
$$

where

$$
\vartheta_{q}(t, x):=F\left(R_{q-1}(t, x)\right) \Theta_{\mu_{q}}\left(\lambda_{q} x\right), \quad w_{q}(t, x):=G\left(R_{q-1}(t, x)\right) W_{\mu_{q}}\left(\lambda_{q} x\right),
$$

where $\lambda_{q}$ is an oscillation parameter and $\mu_{q}$ is a concentration parameter, suitably chosen at each step of iteration, $F, G$ are nonlinear functions and $\left\{\Theta_{\mu}\right\}_{\mu>0}$ (resp. $\left\{W_{\mu}\right\}_{\mu>0}$ ) is a family of Mikado densities (resp. Mikado fields) (see Proposition 5.1 below and in particular estimates (47)).

It is proven in [16] that $\vartheta_{q}, w_{q}$ satisfy the following estimates:

$$
\begin{aligned}
\left\|\vartheta_{q}\right\|_{C_{t} L_{x}^{r^{\prime}}} & \lesssim\left\|R_{q-1}\right\|_{C_{t} L_{x}^{1}}^{1 / r^{\prime}}, \\
\left\|w_{q}\right\|_{C_{t} L_{x}^{r}} & \lesssim\left\|R_{q-1}\right\|_{C_{t} L_{x}^{1}}^{1 / r}, \\
\left\|\vartheta_{q}\right\|_{C_{t} L_{x}^{1}} & \lesssim \mu_{q}^{-\gamma_{1}}, \\
\left\|w_{q}\right\|_{C_{t} L_{x}^{1}} & \lesssim \mu_{q}^{-\gamma_{2}},
\end{aligned}
$$




$$
\left\|D w_{q}\right\|_{C_{t} L_{x}^{p}} \lesssim \lambda_{q} \mu_{q}^{-\gamma_{3}}
$$

where

$$
\begin{aligned}
& \gamma_{1}=(d-1)\left(1-\frac{1}{r^{\prime}}\right), \quad \gamma_{2}=(d-1)\left(1-\frac{1}{r}\right), \\
& \gamma_{3}=(d-1)\left[\frac{1}{r^{\prime}}+\frac{1}{p}-\left(1+\frac{1}{d-1}\right)\right] .
\end{aligned}
$$

Notice that $\gamma_{1}>0$ because $r<\infty$ (and thus $r^{\prime}>1$ ), $\gamma_{2}>0$ because $r>1$ and $\gamma_{3}>0$ because of (9). Estimates (19a), (19b) together with the inductive assumption (14) applied to $R_{q-1}$ guarantee the convergences in (15). Estimate (19e) guarantees the convergence in (16), provided at each step $\mu_{q} \gg \lambda_{q}$, or, more precisely, $\mu_{q}=\lambda_{q}^{c}$, with $c$ any constant satisfying $c>1 / \gamma_{3}$.

A computation then shows that, in order for (13) to be satisfied, $R_{q}$ must be defined as

$$
-R_{q}=\operatorname{div}-1[\underbrace{\operatorname{div}\left(\vartheta_{q} w_{q}-R_{q-1}\right)}_{\text {quadratic term }}+\underbrace{\partial_{t} \vartheta_{q}+\operatorname{div}\left(\vartheta_{q} u_{q-1}\right)+\operatorname{div}\left(\rho_{q-1} w_{q}\right)}_{\text {linear term }}] \text {. }
$$

In order to prove (14), one first uses the oscillation parameter $\lambda_{q}$ to make the (antidivergence of the) quadratic term small. Then, in order to estimate the linear term, one can use concentration. For instance, for the term $\vartheta_{q} u_{q-1}$, we can use (19c)

$$
\left\|\operatorname{div}^{-1}\left(\operatorname{div}\left(\vartheta_{q} u_{q-1}\right)\right)\right\|_{C_{t} L_{x}^{1}}=\left\|\vartheta_{q} u_{q-1}\right\|_{C_{t} L_{x}^{1}} \lesssim\left\|\vartheta_{q}\right\|_{C_{t} L_{x}^{1}} \leq \mu_{q}^{-\gamma_{1}} \leq \delta_{q},
$$

provided $\mu_{q}$ is chosen large enough. A similar estimate holds for $\partial_{t} \vartheta_{q}$, again using (19c), while for $\rho_{q-1} w_{q}$ one must use (19d).

This shows that $R_{q}$ can be suitably defined in order to satisfy (20), thus concluding the proof in [16] for the analog of Theorem 1.1 under the assumptions (8), (9). Let us now discuss why the above proof does not apply to Theorem 1.1, i.e. to the case $r=\infty, r^{\prime}=1$.

\subsection{First issue}

If $r=\infty$, then estimate (19b) becomes $\left\|w_{q}\right\|_{C_{t x}} \lesssim 1$ and this is not enough to prove the convergence in (15b). This issue is solved, modifying the definition of $\rho_{q}, u_{q}$ in (17) as

$$
\rho_{q}:=\rho_{q-1}+\eta_{q} \vartheta_{q}, \quad u_{q}:=u_{q-1}+\frac{1}{\eta_{q}} w_{q},
$$

and choosing $\eta_{q}:=\left\|R_{q-1}\right\|_{C_{t} L_{x}^{1}}^{-1 / 2}$. In this way, using (19a), we get

$$
\left\|\rho_{q}-\rho_{q-1}\right\|_{C_{t} L_{x}^{1}} \lesssim \eta_{q}\left\|R_{q-1}\right\|_{C_{t} L_{x}^{1}} \leq\left\|R_{q-1}\right\|_{C_{t} L_{x}^{1}}^{1 / 2} \leq \delta_{q-1}^{1 / 2}
$$

and

$$
\left\|u_{q}-u_{q-1}\right\|_{C_{t x}} \lesssim \frac{1}{\eta_{q}} \leq\left\|R_{q-1}\right\|_{C_{t} L_{x}^{1}}^{1 / 2} \leq \delta_{q-1}^{1 / 2},
$$

so that the convergences in (15) still holds, and, moreover, the limit vector field $u=\lim u_{q}$ is continuous, being the uniform limit of smooth fields. See Sect. 4 and, in particular, estimates (43), (44). 


\subsection{Second issue}

The second issue concerns the analysis of the linear term in (20) and in particular estimate (21) and the companion estimate for $\partial_{t} \vartheta_{q}$. Indeed, if $r=\infty$ and $r^{\prime}=1$, then $\gamma_{1}=0$ and thus the concentration paramter $\mu_{q}$ can not be used in (21) to make the linear term smaller than $\delta_{q}$.

This issue is solved using the inverse flow map associated to $u_{q-1}$, an idea used in [4] in the framework of the Euler equation, see also [5,13]. Precisely, one separately considers

$$
\text { Linear term in }(20)=\underbrace{\partial_{t} \vartheta_{q}+\operatorname{div}\left(\vartheta_{q} u_{q-1}\right)}_{\text {transport term }}+\underbrace{\operatorname{div}\left(\rho_{q-1} u_{q}\right)}_{\text {Nash term }} .
$$

While for the Nash term an estimate similar to (21) still holds, since $\gamma_{2}=d-1>0$, in order to treat the transport term, one modifies the definition of $\vartheta_{q}$ and $w_{q}$ as follows. The time interval $[0,1]$ is split into $N$ small intervals $\left\{I_{i}\right\}_{i}$ of size $1 / N$. Denoting by $t_{i}$ the middle point of each $I_{i}$, one considers the inverse flow map $\Phi_{i}$ associated to $u_{q-1}$

$$
\left\{\begin{array}{l}
\partial_{t} \Phi_{i}+\left(u_{q-1} \cdot \nabla\right) \Phi_{i}=0 \\
\Phi_{i}\left(t_{i}, x\right)=x
\end{array}\right.
$$

and a partition of unity $\left\{\zeta_{i}\right\}$ subordinated to the partition $\left\{I_{i}\right\}_{i}$ of $[0,1]$. The definition in (18) is then modified as follows:

$$
\begin{aligned}
& \vartheta_{q}(t, x):=F\left(R_{q-1}(t, x)\right) \sum_{i} \zeta_{i}(t) \Theta_{\mu_{q}}\left(\lambda_{q} \Phi_{i}(t, x)\right), \\
& w_{q}(t, x):=G\left(R_{q-1}(t, x)\right) \sum_{i} \zeta_{i}(t) W_{\mu_{q}}\left(\lambda_{q} \Phi_{i}(t, x)\right) .
\end{aligned}
$$

With this new definition, the transport term in (22) assumes the form

$$
\text { Transport term in }(22)=\sum_{i} H_{i}(t, x) \Theta_{\mu_{q}}\left(\lambda_{q} \Phi_{i}(t, x)\right) .
$$

The oscillation parameter $\lambda_{q}$ can now be used to show that

$$
\operatorname{div}^{-1}[\text { Transport term in }(22)] \approx \frac{1}{\lambda_{q}} \lesssim \delta_{q} .
$$

See Sect. 6.3.

\subsection{Third issue}

The third issue appears because of the new definition (23) of $\vartheta_{q}, w_{q}$. Indeed if at some time $t \in[0,1]$ two cutoffs $\zeta_{i}(t) \neq 0, \zeta_{i+1}(t) \neq 0$ are active, then in the quadratic term in (20) a term of the form

$$
\operatorname{div}\left[F\left(R_{q-1}\right) G\left(R_{q-1}\right) \Theta_{\mu_{q}}\left(\lambda_{q} \Phi_{i}(t, x)\right) W_{\mu_{q}}\left(\lambda_{q} \Phi_{i+1}(t, x)\right)\right]
$$

appears, i.e. a non-trivial interaction between a Mikado density and a Mikado field. In general there is no reason why one should be able to find a small antidivergence of such term. The 
problem can be solved, using, at each step $q$ of the construction, two different oscillation parameters $\lambda_{q}^{\prime}, \lambda_{q}^{\prime \prime}$ and two different concentration parameters $\mu_{q}^{\prime}, \mu_{q}^{\prime \prime}$ with

$$
\lambda_{q}^{\prime} \ll \lambda_{q}^{\prime \prime}, \quad \mu_{q}^{\prime} \ll \mu_{q}^{\prime \prime}
$$

and modifying one more time the definition of $\vartheta_{q}, w_{q}$ as follows:

$$
\begin{aligned}
& \vartheta_{q}(t, x)=F\left(R_{q-1}(t, x)\right)\left[\sum_{i \text { odd }} \zeta_{i}(t) \Theta_{\mu_{q}^{\prime}}\left(\lambda_{q}^{\prime} \Phi_{i}(t, x)\right)+\sum_{i \text { even }} \zeta_{i}(t) \Theta_{\mu_{q}^{\prime \prime}}\left(\lambda_{q}^{\prime \prime} \Phi_{i}(t, x)\right)\right], \\
& w_{q}(t, x)=G\left(R_{q-1}(t, x)\right)\left[\sum_{i \text { odd }} \zeta_{i}(t) W_{\mu_{q}^{\prime}}\left(\lambda_{q}^{\prime} \Phi_{i}(t, x)\right)+\sum_{i \text { even }} \zeta_{i}(t) W_{\mu_{q}^{\prime \prime}}\left(\lambda_{q}^{\prime \prime} \Phi_{i}(t, x)\right)\right] .
\end{aligned}
$$

See (55) for the slightly different, precise definition of the perturbations. With this new definition, the main term in the non-trivial interaction in (24) becomes of the form

$$
\Theta_{\mu_{q}^{\prime}}\left(\lambda_{q}^{\prime} \Phi_{i}(t, x)\right) W_{\mu_{q}^{\prime \prime}}\left(\lambda_{q}^{\prime \prime} \Phi_{i+1}(t, x)\right) \text { or } \Theta_{\mu_{q}^{\prime \prime}}\left(\lambda_{q}^{\prime \prime} \Phi_{i}(t, x)\right) W_{\mu_{q}^{\prime}}\left(\lambda_{q}^{\prime} \Phi_{i+1}(t, x)\right)
$$

i.e. the product of a fast oscillating function (with frequency $\lambda_{q}^{\prime}$ ) with a very fast oscillating function (with frequency $\lambda_{q}^{\prime \prime}$ ), where one of the two factors (namely $W_{\mu_{q}^{\prime}}$ or $W_{\mu_{q}^{\prime \prime}}$ ) is small in $L^{1}\left(\mathbb{T}^{d}\right)$ because of the concentration mechanism (compare with estimate (19d)). One can then use an improved Hölder inequality (see Lemma 3.4) to show that the terms in (25) are small in $L^{1}$ and thus conclude the proof of Theorem 1.1. See Sect. 6.2 and in particular Lemma 6.1.

\section{Technical tools}

In this section we provide some technical tools which will be frequently used in the following. We start by fixing some notation:

- $\mathbb{T}^{d}=\mathbb{R}^{d} / \mathbb{Z}^{d}$ is the $d$-dimensional flat torus, $d \geq 3$.

- If $g(x)$ is a smooth function of $x \in \mathbb{T}^{d}$, we denote by $\|g\|_{L^{p}\left(\mathbb{T}^{d}\right)}$, or simply by $\|g\|_{L^{p}}$, its $L^{p}$-norm, for $p \in[1, \infty]$.

- If $f(t, x)$ is a smooth function of $t \in[0,1]$ and $x \in \mathbb{T}^{d}$, we denote by

- $\|f\|_{C^{k}}$ the sup norm of $f$ together with the sup norm of all its derivatives in time and space up to order $k$;

- $\|f(t)\|_{C^{k}\left(\mathbb{T}^{d}\right)}$, or simply $\|f(t)\|_{C^{k}}$, the sup norm of $x \mapsto f(t, x)$ together with the sup norm of all its spatial derivatives up to order $k$ at fixed time $t$;

- $\|f(t)\|_{L^{p}\left(\mathbb{T}^{d}\right)}$, or simply $\|f(t)\|_{L^{p}}$, the $L^{p}$ norm of $f$ in the spatial derivatives, at fixed time $t$.

- If $f:[0,1] \rightarrow \mathbb{R}$ is a function of time only, we will denote by $\dot{f}=d f / d t$ its derivative.

- $C_{0}^{\infty}\left(\mathbb{T}^{d}\right)$ is the set of smooth functions on the torus with zero mean value.

- $\mathbb{N}=\{0,1,2, \ldots\}, \mathbb{N}^{*}=\mathbb{N} \backslash\{0\}$.

- We will use the notation $C\left(A_{1}, \ldots, A_{n}\right)$ to denote a constant which depends only on the numbers $A_{1}, \ldots, A_{n}$. 


\subsection{Diffeomorphisms of the flat torus}

We discuss in this section standard properties of diffeomorphisms of the flat torus. Let $\Phi$ : $\mathbb{R}^{d} \rightarrow \mathbb{R}^{d}$ be a smooth diffeomorphism. We say that $\Phi$ is a diffeomorphism of $\mathbb{T}^{d}$, and we write $\Phi: \mathbb{T}^{d} \rightarrow \mathbb{T}^{d}$, if

$$
\Phi(x+k)=\Phi(x)+k, \quad \text { for every } k \in \mathbb{Z}^{d} .
$$

We say that a diffeomorphism $\Phi: \mathbb{T}^{d} \rightarrow \mathbb{T}^{d}$ is measure-preserving if $|\operatorname{det} D \Phi(x)|=1$ for every $x \in \mathbb{T}^{d}$. Given a diffeomorphism $\Phi$, we will often consider

(1) the derivative $D \Phi: \mathbb{T}^{d} \rightarrow \mathbb{R}^{d \times d}$;

(2) the inverse-matrix of the derivative $(D \Phi)^{-1}: \mathbb{T}^{d} \rightarrow \mathbb{R}^{d \times d}$;

(3) higher order derivatives of the inverse-matrix of the derivative $D^{k}\left((D \Phi)^{-1}\right): \mathbb{T}^{d} \rightarrow$ $\mathbb{R}^{d(k+2)}$.

Observe that, given a matrix $A \in \mathbb{R}^{d \times d}$, with $|\operatorname{det} A|=1$, it holds $|A| \geq 1$, where $|A|:=\max _{|u|=1}|A u|$ is the norm of matrix $A$. Therefore if $\Phi$ is a measure-preserving diffeomorphism, then $|D \Phi(x)| \geq 1$ for every $x \in \mathbb{T}^{d}$ and thus $1 \leq\|D \Phi\|_{C^{k}}^{\alpha} \leq\|D \Phi\|_{C^{k}}^{\beta}$ for every $0<\alpha<\beta$. Recall also that for a given invertible matrix $A$,

$$
A^{-1}=\frac{1}{\operatorname{det} A}(\operatorname{cof} A)^{T},
$$

where $(\operatorname{cof} A)^{T}$ is transpose of the cofactor matrix of $A$.

Lemma 3.1 Let $\Phi: \mathbb{T}^{d} \rightarrow \mathbb{T}^{d}$ be a measure-preserving smooth diffeomorphism. Then, for every $k \in \mathbb{N}$,

$$
\left\|D^{k}\left((D \Phi)^{-1}\right)\right\|_{C^{0}\left(\mathbb{T}^{d}\right)} \leq C_{k}\|D \Phi\|_{C^{k}\left(\mathbb{T}^{d}\right)}^{d-1},
$$

where $C_{k}$ is a constant depending only on $k$ (and on the dimension $d$ ).

Proof. For any fixed $x \in \mathbb{T}^{d}$ it holds

$$
\left|[D \Phi(x)]^{-1}\right|=\left|\frac{1}{\operatorname{det} D \Phi(x)}(\operatorname{cof} D \Phi(x))^{T}\right|=|\operatorname{cof} D \Phi(x)| .
$$

The conclusion now follows from the definition of cofactor matrix.

Lemma 3.2 Let $G: \mathbb{T}^{d} \rightarrow \mathbb{R}^{d}, g: \mathbb{T}^{d} \rightarrow \mathbb{R}$ be smooth and assume div $G=g$. Let $\Phi: \mathbb{T}^{d} \rightarrow \mathbb{T}^{d}$ be a measure-preserving diffeomorphism of the torus. Then

$$
\operatorname{div}\left[(D \Phi)^{-1} G(\Phi)\right]=g(\Phi)
$$

Proof. We show that for every $\varphi \in C^{\infty}\left(\mathbb{T}^{d}\right)$ it holds

$$
\int_{\mathbb{T}^{d}} \varphi \operatorname{div}\left[(D \Phi)^{-1} G(\Phi)\right] d x=\int_{\mathbb{T}^{d}} \varphi g(\Phi) d x .
$$


Set $\tilde{\varphi}:=\varphi \circ \Phi^{-1}$. It holds

$$
\begin{aligned}
\int_{\mathbb{T}^{d}} \varphi \operatorname{div}\left[(D \Phi)^{-1} G(\Phi)\right] d x & =\int_{\mathbb{T}^{d}} \tilde{\varphi}(\Phi) \operatorname{div}\left[(D \Phi)^{-1} G(\Phi)\right] d x \\
& =-\int_{\mathbb{T}^{d}}\left[(D \Phi)^{T} \nabla \tilde{\varphi}(\Phi)\right] \cdot\left[(D \Phi)^{-1} G(\Phi)\right] d x \\
& =-\int_{\mathbb{T}^{d}} \nabla \tilde{\varphi}(\Phi) \cdot G(\Phi) d x \\
\text { (changing variable } y=\Phi(x)) & =-\int_{\mathbb{T}^{d}} \nabla \tilde{\varphi} \cdot G d y \\
& =\int_{\mathbb{T}^{d}} \tilde{\varphi} \operatorname{div} G d y \\
& =\int_{\mathbb{T}^{d}} \tilde{\varphi} g d y \\
& =\int_{\mathbb{T}^{d}} \varphi\left(\Phi^{-1}\right) g d y \\
\text { (changing variable } \left.x=\Phi^{-1}(y)\right) & =\int_{\mathbb{T}^{d}} \varphi g(\Phi) d x
\end{aligned}
$$

thus concluding the proof of the lemma.

Lemma 3.3 Let $g: \mathbb{T}^{d} \rightarrow \mathbb{R}$ be a smooth function. Let $\Phi: \mathbb{T}^{d} \rightarrow \mathbb{T}^{d}$ be a measurepreserving diffeomorphism. Then for every $p \in[1, \infty]$ and $k \in \mathbb{N}, k \geq 1$,

$$
\|g \circ \Phi\|_{L^{p}\left(\mathbb{T}^{d}\right)}=\|g\|_{L^{p}\left(\mathbb{T}^{d}\right)},
$$

and

$$
\|g \circ \Phi\|_{W^{k, p}\left(\mathbb{T}^{d}\right)} \leq C_{k}\|D \Phi\|_{C^{k-1}\left(\mathbb{T}^{d}\right)}^{k}\|g\|_{W^{k, p}\left(\mathbb{T}^{d}\right)} .
$$

The proof is an easy application of the chain rule and thus it is omitted.

\subsection{Properties of fast oscillations}

We discuss now some properties of fast oscillating periodic functions. For a given $g: \mathbb{T}^{d} \rightarrow \mathbb{R}$ and $\lambda \in \mathbb{N}^{*}$, we set

$$
g_{\lambda}(x):=g(\lambda x) .
$$

Observe that for every $p \in[1, \infty]$ and $k \in \mathbb{N}$,

$$
\left\|D^{k} g_{\lambda}\right\|_{L^{p}\left(\mathbb{T}^{d}\right)}=\lambda^{k}\left\|D^{k} g\right\|_{L^{p}\left(\mathbb{T}^{d}\right)} .
$$

\subsubsection{Improved Hölder inequality}

In the same spirit as in [16] and [6], we now prove an improved Hölder inequality for the product of a slow oscillating function with a fast oscillating functions composed with a diffeomorphism. 
Lemma 3.4 (Improved Hölder inequality). Let $f, g: \mathbb{T}^{d} \rightarrow \mathbb{R}$ be smooth functions, $\lambda \in \mathbb{N}^{*}$ and $\Phi: \mathbb{T}^{d} \rightarrow \mathbb{T}^{d}$ be a measure-preserving diffeomorphism. Then for every $p \in[1, \infty]$,

$$
\left\|f g_{\lambda}\right\|_{L^{p}\left(\mathbb{T}^{d}\right)} \leq\|f\|_{L^{p}\left(\mathbb{T}^{d}\right)}\|g\|_{L^{p}\left(\mathbb{T}^{d}\right)}+\frac{C_{p}}{\lambda^{1 / p}}\|f\|_{C^{1}\left(\mathbb{T}^{d}\right)}\|g\|_{L^{p}\left(\mathbb{T}^{d}\right)}
$$

and

$$
\begin{aligned}
\left\|f \cdot\left(g_{\lambda} \circ \Phi\right)\right\|_{L^{p}\left(\mathbb{T}^{d}\right)} \leq & \|f\|_{L^{p}\left(\mathbb{T}^{d}\right)}\|g\|_{L^{p}\left(\mathbb{T}^{d}\right)} \\
& +\frac{C_{p}}{\lambda^{1 / p}}\|f\|_{C^{1}\left(\mathbb{T}^{d}\right)}\|D \Phi\|_{C^{0}\left(\mathbb{T}^{d}\right)}^{d-1}\|g\|_{L^{p}\left(\mathbb{T}^{d}\right)} .
\end{aligned}
$$

Here $f \cdot\left(g_{\lambda} \circ \Phi\right)$ is the function $x \mapsto f(x) g(\lambda \Phi(x))$.

Proof. For a proof of (29), see [16, Lemma 2.1]. Concerning (30), we argue as follows. Since $\Phi$ is a measure-preserving diffeomorphism, it holds

$$
\left\|f \cdot\left(g_{\lambda} \circ \Phi\right)\right\|_{L^{p}}=\left\|\left(f \circ \Phi^{-1}\right) \cdot g_{\lambda}\right\|_{L^{p}} .
$$

Therefore we can apply (29) to get

$$
\left\|f \cdot\left(g_{\lambda} \circ \Phi\right)\right\|_{L^{p}} \leq\left\|f \circ \Phi^{-1}\right\|_{L^{p}}\|g\|_{L^{p}}+\frac{C_{p}}{\lambda^{1 / p}}\left\|f \circ \Phi^{-1}\right\|_{C^{1}}\|g\|_{L^{p}}
$$

(by Lemma 3.3 and $(28)) \leq\|f\|_{L^{p}}\|g\|_{L^{p}}+\frac{C_{p}}{\lambda^{1 / p}}\|f\|_{C^{1}}\left\|D\left(\Phi^{-1}\right)\right\|_{C^{0}}\|g\|_{L^{p}}$

$$
\leq\|f\|_{L^{p}}\|g\|_{L^{p}}+\frac{C_{p}}{\lambda^{1 / p}}\|f\|_{C^{1}}\left\|(D \Phi)^{-1}\right\|_{C^{0}}\|g\|_{L^{p}}
$$

(by Lemma 3.1) $\leq\|f\|_{L^{p}}\|g\|_{L^{p}}+\frac{C_{p}}{\lambda^{1 / p}}\|f\|_{C^{1}}\|D \Phi\|_{C^{0}}^{d-1}\|g\|_{L^{p}}$.

\subsubsection{Antidivergence operators}

In this section we introduce two antidivergence operators, a standard and an improved one, in the same spirit as in [16].

For $f \in C_{0}^{\infty}\left(\mathbb{T}^{d}\right)$ there exists a unique $u \in C_{0}^{\infty}\left(\mathbb{T}^{d}\right)$ such that $\Delta u=f$. The operator $\Delta^{-1}: C_{0}^{\infty}\left(\mathbb{T}^{d}\right) \rightarrow C_{0}^{\infty}\left(\mathbb{T}^{d}\right)$ is thus well defined. We define the standard antidivergence operator as $\nabla \Delta^{-1}: C_{0}^{\infty}\left(\mathbb{T}^{d}\right) \rightarrow C^{\infty}\left(\mathbb{T}^{d} ; \mathbb{R}^{d}\right)$. It clearly satisfies $\operatorname{div}\left(\nabla \Delta^{-1} f\right)=f$.

Lemma 3.5 For every $k \in \mathbb{N}$ and $p \in[1, \infty]$, the standard antidivergence operator satisfies the bounds

$$
\left\|D^{k}\left(\nabla \Delta^{-1} g\right)\right\|_{L^{p}\left(\mathbb{T}^{d}\right)} \leq C_{k, p}\left\|D^{k} g\right\|_{L^{p}\left(\mathbb{T}^{d}\right)} .
$$

For the proof, see [16, Lemma 2.2] .

We now introduce an improved antidivergence operator, which allows us to get better (w.r.t $\nabla \Delta^{-1}$ ) estimates, if applied to the product of a slow oscillating function with a fast oscillating one composed with a diffeomorphism.

Lemma 3.6 Let $f, g: \mathbb{T}^{d} \rightarrow \mathbb{R}$ be smooth function with

$$
f g=0 .
$$


Let $\lambda \in \mathbb{N}^{*}$ and $\Phi: \mathbb{T}^{d} \rightarrow \mathbb{T}^{d}$ be a smooth, measure-preserving diffeomorphism. Then there exists a smooth vector field $u: \mathbb{T}^{d} \rightarrow \mathbb{R}^{d}$ so that

$$
\operatorname{div} u=f \cdot\left(g_{\lambda} \circ \Phi\right)-f f \cdot\left(g_{\lambda} \circ \Phi\right)
$$

and for every $k \in \mathbb{N}, p \in[1, \infty]$,

$$
\|u\|_{W^{k, p}\left(\mathbb{T}^{d}\right)} \leq C_{k, p} \lambda^{k-1}\|f\|_{C^{k+1}\left(\mathbb{T}^{d}\right)}\|D \Phi\|_{C^{k}\left(\mathbb{T}^{d}\right)}^{d-1+k}\|g\|_{W^{k, p}\left(\mathbb{T}^{d}\right)} .
$$

We will use the notation

$$
u:=\mathcal{R}\left(f \cdot\left(g_{\lambda} \circ \Phi\right)-f f \cdot\left(g_{\lambda} \circ \Phi\right)\right) .
$$

Remark 3.7 The same result holds if $f, g$ are vector fields and - in (32) denotes the scalar product.

Proof. Since $g$ has zero mean value, we can define

$$
G:=\nabla \Delta^{-1} g .
$$

Let us denote by $H: \mathbb{T}^{d} \rightarrow \mathbb{R}^{d}$ the vector field

$$
H:=\frac{1}{\lambda}(D \Phi)^{-1} G_{\lambda}(\Phi) \text {. }
$$

By Lemma 3.2 it holds

$$
\operatorname{div} H=\frac{1}{\lambda}\left(\operatorname{div} G_{\lambda}\right)(\Phi)=g_{\lambda}(\Phi) .
$$

We now set

$$
u:=f H-\nabla \Delta^{-1}(\nabla f \cdot H-f \nabla f \cdot H) .
$$

Let us first check that $u$ satisfies (32). It holds

$$
\begin{aligned}
\operatorname{div} u & =f \operatorname{div} H+\nabla f \cdot H-\nabla f \cdot H+f \nabla f \cdot H \\
& =f \operatorname{div} H+f \nabla f \cdot H \\
\text { (integrating by parts) } & =f \operatorname{div} H-f f \operatorname{div} H \\
\text { (by (35)) } & =f \cdot\left(g_{\lambda} \circ \Phi\right)-f f \cdot\left(g_{\lambda} \circ \Phi\right) .
\end{aligned}
$$

We prove now that (33) holds. We first estimate $H$ as follows:

$$
\begin{aligned}
\|H\|_{W^{k, p}} & \leq \frac{1}{\lambda}\left\|(D \Phi)^{-1}\right\|_{C^{k}}\left\|G_{\lambda} \circ \Phi\right\|_{W^{k, p}} \\
\text { (by Lemma 3.1) } & \leq \frac{C_{k}}{\lambda}\|D \Phi\|_{C^{k}}^{d-1}\left\|G_{\lambda} \circ \Phi\right\|_{W^{k, p}} \\
\text { (by Lemma 3.3) } & \leq \frac{C_{k}}{\lambda}\|D \Phi\|_{C^{k}}^{d-1+k}\left\|G_{\lambda}\right\|_{W^{k, p}}
\end{aligned}
$$




$$
\begin{aligned}
\text { (by (28)) } & \leq C_{k} \lambda^{k-1}\|D \Phi\|_{C^{k}}^{d-1+k}\|G\|_{W^{k, p}} \\
(\text { by Lemma 3.5) } & \leq C_{k, p} \lambda^{k-1}\|D \Phi\|_{C^{k}}^{d-1+k}\|g\|_{W^{k, p}} .
\end{aligned}
$$

Using now again Lemma 3.5, we can write

$$
\begin{aligned}
\|u\|_{W^{k, p}} & \leq\|f H\|_{W^{k, p}}+C_{k, p}\|\nabla f \cdot H\|_{W^{k, p}} \\
& \leq\|f\|_{C^{k}}\|H\|_{W^{k, p}}+C_{k, p}\|\nabla f\|_{C^{k}}\|H\|_{W^{k, p}} \\
& \leq C_{k, p}\|f\|_{C^{k+1}}\|H\|_{W^{k, p}} \\
& \leq C_{k, p} \lambda^{k-1}\|f\|_{C^{k+1}}\|D \Phi\|_{C^{k}}^{d-1+k}\|g\|_{W^{k, p}},
\end{aligned}
$$

which is what we wanted to prove.

Remark 3.8 In Lemma 3.6, if $f, g, \Phi$ are smooth functions of $(t, x), t \in[0,1], x \in \mathbb{T}^{d}$ and at each time $t \in[0,1]$, they satisfy the assumptions of Lemma 3.6, then we can apply $\mathcal{R}$ at each time and define a time-dependent vector field $u(t, \cdot)$ satisfying (32) and (33). Moreover $u$ turns out to be a smooth function of $(t, x)$.

\subsubsection{Mean value and fast oscillations}

In this section we prodide an estimate on the mean value of the product of a slow oscillating function with a fast oscillating function composed with a diffeomorphism.

Lemma 3.9 Let $f, g: \mathbb{T}^{d} \rightarrow \mathbb{R}$, with $f_{\mathbb{T}^{d}} g=0$. Let $\lambda \in \mathbb{N}^{*}$ and $\Phi: \mathbb{T}^{d} \rightarrow \mathbb{T}^{d}$ be a measure-preserving diffeomorphism. Then

$$
\left|f_{\mathbb{T}^{d}} f g_{\lambda} d x\right| \leq \frac{\sqrt{d}\|f\|_{C^{1}\left(\mathbb{T}^{d}\right)}\|g\|_{L^{1}\left(\mathbb{T}^{d}\right)}}{\lambda}
$$

and

$$
\left|f_{\mathbb{T}^{d}} f \cdot\left(g_{\lambda} \circ \Phi\right) d x\right| \leq \frac{\sqrt{d}\|f\|_{C^{1}\left(\mathbb{T}^{d}\right)}\|D \Phi\|_{C^{0}\left(\mathbb{T}^{d}\right)}^{d-1}\|g\|_{L^{1}\left(\mathbb{T}^{d}\right)}}{\lambda} .
$$

Proof of Theorem 1.1 assuming Theorem 1.2 For a proof of (36), see [16, Lemma 2.6]. The proof of (37) follows from (36), observing that

$$
f f(x) g(\lambda \Phi(x)) d x=f f\left(\Phi^{-1}(y)\right) g(\lambda y) d y .
$$

\section{Statement of the main proposition and proof of Theorem 1.2}

We assume without loss of generality $\mathbb{T}^{d}$ is the periodic extension of the unit cube $[0,1]^{d}$. The following proposition contains the key facts used to prove Theorem 1.2. Let us first introduce the continuity-defect equation:

$$
\left\{\begin{array}{l}
\partial_{t} \rho+\operatorname{div}(\rho u)=-\operatorname{div} R \\
\operatorname{div} u=0 .
\end{array}\right.
$$

We will call $R$ the defect field. 
Proposition 4.1 There exists a constant $M>0$ such that the following holds. Let $p \in$ $[1, d-1), \eta, \delta>0$ and let $\left(\rho_{0}, u_{0}, R_{0}\right)$ be a smooth solution of the continuity-defect equation (38). Then there exists another smooth solution $\left(\rho_{1}, u_{1}, R_{1}\right)$ of (38) such that for every $t \in[0,1]$,

$$
\begin{aligned}
\left\|\rho_{1}(t)-\rho_{0}(t)\right\|_{L^{1}\left(\mathbb{T}^{d}\right)} & \leq M \eta\left\|R_{0}(t)\right\|_{L^{1}\left(\mathbb{T}^{d}\right)}, \\
\left\|u_{1}(t)-u_{0}(t)\right\|_{C^{0}\left(\mathbb{T}^{d}\right)} & \leq M \eta^{-1}, \\
\left\|u_{1}(t)-u_{0}(t)\right\|_{W^{1, p}\left(\mathbb{T}^{d}\right)} & \leq \delta \\
\left\|R_{1}(t)\right\|_{L^{1}\left(\mathbb{T}^{d}\right)} & \leq \delta
\end{aligned}
$$

and, moreover, if at some time $t \in[0,1], R_{0}(t)=0$, then

$$
\rho_{1}(t)-\rho_{0}(t)=u_{1}(t)-u_{0}(t)=R_{1}(t)=0 .
$$

Proof of Theorem 1.2 assuming Proposition 4.1 For $\rho_{0}, u_{0}$ in the statement of Theorem 1.2, define

$$
R_{0}(t):=-\nabla \Delta^{-1}\left(\partial_{t} \rho_{0}(t)+\operatorname{div}\left(\rho_{0}(t) u_{0}(t)\right)\right) .
$$

By (11), $R_{0}$ is well defined, it is smooth and $\left(\rho_{0}, u_{0}, R_{0}\right)$ solve the continuity-defect equation.

Let $\left(p_{q}\right)_{q \in \mathbb{N}}$ be a fixed increasing sequence of real numbers such that $p_{q} \rightarrow d-1$ as $q \rightarrow \infty$. Let also $\left(\eta_{q}\right)_{q \in \mathbb{N}},\left(\delta_{q}\right)_{q \in \mathbb{N}}$ be two sequence of positive real numbers, which will be fixed later. Starting from $\left(\rho_{0}, u_{0}, R_{0}\right)$, we can recursively apply Proposition 4.1 to obtain a sequence $\left(\rho_{q}, u_{q}, R_{q}\right)_{q \in \mathbb{N}}$ of smooth solutions to the continuity-defect equation such that

$$
\begin{aligned}
& \left\|\rho_{q+1}(t)-\rho_{q}(t)\right\|_{L^{1}\left(\mathbb{T}^{d}\right)} \leq M \eta_{q}\left\|R_{q}(t)\right\|_{L^{1}\left(\mathbb{T}^{d}\right)}, \\
& \left\|u_{q+1}(t)-u_{q}(t)\right\|_{C^{0}\left(\mathbb{T}^{d}\right)} \leq M \eta_{q}^{-1}, \\
& \left\|u_{q+1}(t)-u_{q}(t)\right\|_{W^{1, p_{q}\left(\mathbb{T}^{d}\right)}} \leq \delta_{q}, \\
& \left\|R_{q+1}(t)\right\|_{L^{1}\left(\mathbb{T}^{d}\right)} \leq \delta_{q},
\end{aligned}
$$

for all times $t \in[0,1]$ and

$$
\rho_{q+1}(t)=\rho_{q}(t), \quad u_{q+1}(t)=u_{q}(t), \quad R_{q+1}(t)=0,
$$

for all times $t$ such that $R_{q}(t)=0$. Therefore, by induction, we get from (40a) and (40d) that for all $t \in[0,1]$ and all $q \in \mathbb{N}$,

$$
\left\|\rho_{q+1}(t)-\rho_{q}(t)\right\|_{L^{1}\left(\mathbb{T}^{d}\right)} \leq M \eta_{q} \delta_{q-1},
$$

where we set $\delta_{-1}:=\max _{t \in[0,1]}\left\|R_{0}(t)\right\|_{L^{1}}$ and, moreover,

$$
\rho_{q+1}(t)=\rho_{q}(t), \quad u_{q+1}(t)=u_{q}(t) \quad \text { for all } t \in E,
$$

where $E$ was defined in (12). We now choose $\left(\delta_{q}\right)_{q \in \mathbb{N}}$ so that

$$
\sum_{q=0}^{+\infty} \delta_{q}<\sum_{q=0}^{+\infty} \delta_{q}^{1 / 2}<\infty
$$

and

$$
\eta_{q}:=\sigma \delta_{q-1}^{-1 / 2}
$$


for $q \in \mathbb{N}$, where $\sigma>0$ is a positive number, to be defined later. From (41) we get, for all $t \in[0,1]$,

$$
\sum_{q=0}^{+\infty}\left\|\rho_{q+1}(t)-\rho_{q}(t)\right\|_{L^{1}\left(\mathbb{T}^{d}\right)} \leq M \sum_{q=0}^{+\infty} \eta_{q} \delta_{q-1}=M \sigma \sum_{q=0}^{+\infty} \delta_{q-1}^{1 / 2}<\infty,
$$

and thus there exists $\rho \in C\left([0,1] ; L^{1}\left(\mathbb{T}^{d}\right)\right)$ so that $\rho_{q} \rightarrow \rho$ in $C\left([0,1] ; L^{1}\left(\mathbb{T}^{d}\right)\right)$. Similarly, using (40b), for all $t \in[0,1]$,

$$
\sum_{q=0}^{+\infty}\left\|u_{q+1}(t)-u_{q}(t)\right\|_{C^{0}\left(\mathbb{T}^{d}\right)} \leq M \sum_{q=0}^{+\infty} \eta_{q}^{-1}=M \sigma^{-1} \sum_{q=0}^{+\infty} \delta_{q-1}^{1 / 2}<\infty,
$$

and thus there exists $u \in C\left([0,1] \times \mathbb{T}^{d} ; \mathbb{R}^{d}\right)$ so that $u_{q} \rightarrow u$ uniformly. It follows now from (40d) that $\rho, u$ solve (1).

To prove that $u \in \bigcap_{1 \leq p<d-1} C_{t} W_{x}^{1, p}$, fix $p \in[1, d-1)$. There is $q^{*}$ so that $p_{q}>p$ for every $q>q^{*}$. We now have, for all $t \in[0,1]$,

$$
\begin{aligned}
\sum_{q=0}^{+\infty}\left\|u_{q+1}(t)-u_{q}(t)\right\|_{W^{1, p}\left(\mathbb{T}^{d}\right)}= & \sum_{q=0}^{q^{*}}\left\|u_{q+1}(t)-u_{q}(t)\right\|_{W^{1, p}\left(\mathbb{T}^{d}\right)} \\
& +\sum_{q=q^{*}+1}^{+\infty}\left\|u_{q+1}(t)-u_{q}(t)\right\|_{W^{1, p}\left(\mathbb{T}^{d}\right)} \\
\left(\text { since } p<p_{q} \text { for } q>q^{*}\right) \leq & \sum_{q=0}^{q^{*}}\left\|u_{q+1}(t)-u_{q}(t)\right\|_{W^{1, p}\left(\mathbb{T}^{d}\right)} \\
& +\sum_{q=q^{*}+1}^{+\infty}\left\|u_{q+1}(t)-u_{q}(t)\right\|_{W^{1, p} q}\left(\mathbb{T}^{d}\right) \\
\text { (by (40c) }) \leq & \sum_{q=0}^{q^{*}}\left\|u_{q+1}(t)-u_{q}(t)\right\|_{W^{1, p}\left(\mathbb{T}^{d}\right)}+\sum_{q=q^{*}+1}^{+\infty} \delta_{q}<\infty,
\end{aligned}
$$

thus proving that $u \in C\left([0,1] ; W^{1, p}\left(\mathbb{T}^{d}\right)\right)$. This concludes the proof of parts (a), (b) in the statement of Theorem 1.2.

It follows from (42) that $\rho(t)=\rho_{0}(t)$ and $u(t)=u_{0}(t)$, whenever $t \in E$, and thus part (c) is also proven. To prove (d), we observe that, from (43), for all $t \in[0,1]$,

$$
\left\|\rho(t)-\rho_{0}(t)\right\|_{L^{1}\left(\mathbb{T}^{d}\right)} \leq \sum_{q=0}^{+\infty}\left\|\rho_{q+1}(t)-\rho_{q}(t)\right\|_{L^{1}\left(\mathbb{T}^{d}\right)} \leq M \sigma \sum_{q=0}^{\infty} \delta_{q-1}^{1 / 2}
$$

and thus (d) follows choosing

$$
\sigma:=\frac{\varepsilon}{M \sum_{q=0}^{+\infty} \delta_{q-1}^{1 / 2}}
$$

Alternatively, to achieve (d'), we observe that, from (44), for all $t \in[0,1]$,

$$
\left\|u-u_{0}\right\|_{C^{0}} \leq M \sigma^{-1} \sum_{q=0}^{+\infty} \delta_{q-1}^{1 / 2}
$$


and thus (d)' follows choosing

$$
\sigma:=\frac{M \sum_{q=0}^{+\infty} \delta_{q-1}^{1 / 2}}{\varepsilon} .
$$

Remark 4.2 Estimate (39a), with $\eta$ in the r.h.s., and estimate (39b), with $\eta^{-1}$ in the r.h.s. show that conditions (d)-(d') in the statement of Theorem 1.2 can not be simultaneously achieved.

\section{The perturbations}

In this and the next two sections we prove Proposition 4.1. In particular in this section we fix the constant $M$ in the statement of the proposition, we define the functions $\rho_{1}$ and $u_{1}$ and we estimate them. In Sect. 6 we define $R_{1}$ and we estimate it. In Sect. 7 we conclude the proof of Proposition 4.1.

\subsection{Mikado fields and Mikado densities}

We recall the following proposition from [16].

Proposition 5.1 Let $a, b \in \mathbb{R}$ with

$$
a+b=d-1 \text {. }
$$

For every $\mu>2 d$ and $j=1, \ldots, d$ there exist a Mikado density $\Theta_{\mu}^{j}: \mathbb{T}^{d} \rightarrow \mathbb{R}$ and $a$ Mikado field $W_{\mu}^{j}: \mathbb{T}^{d} \rightarrow \mathbb{R}^{d}$ with the following properties.

(a) It holds

$$
\left\{\begin{array}{l}
\operatorname{div} W_{\mu}^{j}=0, \\
\operatorname{div}\left(\Theta_{\mu}^{j} W_{\mu}^{j}\right)=0, \\
f_{\mathbb{T}^{d}} \Theta_{\mu}^{j}=f_{\mathbb{T}^{d}} W_{\mu}^{j}=0, \\
f_{\mathbb{T}^{d}} \Theta_{\mu}^{j} W_{\mu}^{j}=e_{j},
\end{array}\right.
$$

where $\left\{e_{j}\right\}_{j=1, \ldots, d}$ is the standard basis in $\mathbb{R}^{d}$.

(b) For every $k \in \mathbb{N}$ and $r \in[1, \infty]$

$$
\begin{aligned}
\left\|D^{k} \Theta_{\mu}^{j}\right\|_{L^{r}\left(\mathbb{T}^{d}\right)} & \leq M_{k} \mu^{a+k-(d-1) / r}, \\
\left\|D^{k} W_{\mu}^{j}\right\|_{L^{r}\left(\mathbb{T}^{d}\right)} & \leq M_{k} \mu^{b+k-(d-1) / r},
\end{aligned}
$$

where $M_{k}$ is a constant which depends only on $k$, but not on $r$ and $\mu$.

(c) For $j \neq k$, supp $\Theta_{\mu}^{j}=\operatorname{supp} W_{\mu}^{j}$ and supp $\Theta_{\mu}^{j} \cap \operatorname{supp} W_{\mu}^{k}=\emptyset$.

We now define the constant $M$ in the statement of Proposition 4.1 as

$$
M:=4 d \max \left\{M_{0}, M_{0}^{2}, M_{0}+M_{1}\right\}
$$

and we choose

$$
a:=d-1, \quad b:=0,
$$


in Proposition 5.1. In this way for each direction $j=1, \ldots, d$, we obtain a family of Mikado densities $\left\{\Theta_{\mu}^{j}\right\}_{\mu>2 d}$ and fields $\left\{W_{\mu}^{j}\right\}_{\mu>2 d}$, obeying the following estimates:

$$
\sum_{j=1}^{d}\left\|\Theta_{\mu}^{j}\right\|_{L^{1}\left(\mathbb{T}^{d}\right)}, \sum_{j=1}^{d}\left\|W_{\mu}^{j}\right\|_{L^{\infty}\left(\mathbb{T}^{d}\right)}, \sum_{j=1}^{d}\left\|\Theta_{\mu}^{j} W_{\mu}^{j}\right\|_{L^{1}\left(\mathbb{T}^{d}\right)} \leq \frac{M}{4},
$$

and

$$
\left\|W_{\mu}^{j}\right\|_{L^{1}\left(\mathbb{T}^{d}\right)} \leq M \mu^{-(d-1)}, \quad\left\|W_{\mu}^{j}\right\|_{W^{1, p}\left(\mathbb{T}^{d}\right)} \leq M \mu^{1-(d-1) / p},
$$

and

$$
\left\|\Theta_{\mu}^{j}\right\|_{C^{1}\left(\mathbb{T}^{d}\right)} \leq M \mu^{d}, \quad\left\|W_{\mu}^{j}\right\|_{C^{1}\left(\mathbb{T}^{d}\right)} \leq M \mu
$$

\subsection{Definition of the perturbations}

We are now in a position to define $\rho_{1}, u_{1}$. The constant $M$ has already been fixed in (48). Let thus $p \in[1, d-1), \eta, \delta>0$ and $\left(\rho_{0}, u_{0}, R_{0}\right)$ be a smooth solution to the continuity-defect equation (38).

Let

$$
\begin{array}{ll}
\tau \in 1 / \mathbb{N}^{*} & \text { "time scale" } \\
\lambda^{\prime}, \lambda^{\prime \prime} \in \mathbb{N} & \text { "oscillation" } \\
\mu^{\prime}, \mu^{\prime \prime}>2 d & \text { "concentration" }
\end{array}
$$

be parameters, which will be fixed later. Set

$$
N:=1 / \tau \in \mathbb{N}^{*}
$$

For every $i=1,2, \ldots, N$, let $I_{i}:=[i \tau,(i+1) \tau]$ and let $t_{i}:=(i+1 / 2) \tau$ be the midpoint of $I_{i}$. Consider a partition of unity $\left\{\zeta_{i}\right\}_{i=1, \ldots, N}$ subordinate to the family of intervals $\left\{I_{i}\right\}_{i=1, \ldots, N}$. More precisely, for every $i=1, \ldots, N, \zeta_{i} \in C^{\infty}([0,1])$ and

- $\operatorname{supp} \zeta_{i} \in[(i-1 / 3) \tau,(i+1+1 / 3) \tau]$;

- $\zeta_{i}(t) \in[0,1]$ for every $t \in[0,1]$;

- $\sum_{i=1}^{N} \zeta_{i}^{2}(t)=1$ for every $t \in[0,1]$.

Notice that for every time $t \in[0,1]$ there is at most one odd index $i_{1}$ and one even index $i_{2}$ so that $\zeta_{i}(t)=0$ for every $i \neq i_{1}, i_{2}$. For every $i=1, \ldots, N$, let $\Phi_{i}:[0,1] \times \mathbb{T}^{d} \rightarrow \mathbb{T}^{d}$ be the solution to

$$
\left\{\begin{array}{l}
\partial_{t} \Phi_{i}+\left(u_{0} \cdot \nabla\right) \Phi_{i}=0 \\
\Phi_{i}\left(t_{i}, x\right)=x
\end{array}\right.
$$

i.e. the inverse flow map associated to the vector field $u_{0}$, starting at time $t_{i}$. Notice that, for fixed $t, \Phi_{i}(t): \mathbb{T}^{d} \rightarrow \mathbb{T}^{d}$ is a measure-preserving diffeomorphism.

We denote by $R_{0, j}$ the components of $R_{0}$, i.e.

$$
R_{0}(t, x):=\sum_{j=1}^{d} R_{0, j}(t, x) e_{j} .
$$


Let also $\psi:[0,1] \rightarrow \mathbb{R}$ be a smooth function such that $\psi(t) \in[0,1]$ for every $t \in[0,1]$ and

$$
\psi(t)= \begin{cases}0, & \text { if }\left\|R_{0}(t)\right\|_{L^{1}\left(\mathbb{T}^{d}\right) \leq \delta / 8} \\ 1, & \text { if }\left\|R_{0}(t)\right\|_{L^{1}\left(\mathbb{T}^{d}\right) \geq \delta / 4}\end{cases}
$$

We set

$$
\rho_{1}:=\rho_{0}+\vartheta+\vartheta_{c}, \quad u_{1}:=u_{0}+w,
$$

where $\vartheta, \vartheta_{c}, w$ are defined as follows. First of all, let $\Theta_{\mu}^{j}, W_{\mu}^{j}, j=1, \ldots, d$, be the family (depending on $\mu$ ) of Mikado densities and fields provided by Proposition 5.1, with $a, b$ chosen as in (49). We set

$$
\begin{aligned}
\vartheta(t, x):= & \eta \psi(t)\left\{\sum_{\substack{i=1 \\
i \text { odd }}}^{N} \zeta_{i}(t) \sum_{j=1}^{d} R_{0, j}(t, x) \Theta_{\mu^{\prime}}^{j}\left(\lambda^{\prime} \Phi_{i}(t, x)\right)\right. \\
& \left.+\sum_{\substack{i=1 \\
i \text { even }}}^{N} \zeta_{i}(t) \sum_{j=1}^{d} R_{0, j}(t, x) \Theta_{\mu^{\prime \prime}}^{j}\left(\lambda^{\prime \prime} \Phi_{i}(t, x)\right)\right\}, \\
w(t, x):= & \frac{\psi(t)}{\eta}\left\{\sum_{i=1}^{N} \zeta_{i}(t) \sum_{j=1}^{d}\left(D \Phi_{i}(t, x)\right)^{-1} W_{\mu^{\prime}}^{j}\left(\lambda^{\prime} \Phi_{i}(t, x)\right)\right. \\
& \left.+\sum_{\substack{i=1 \\
i \text { odd }}}^{N} \zeta_{i}(t) \sum_{j=1}^{d}\left(D \Phi_{i}(t, x)\right)^{-1} W_{\mu^{\prime \prime}}^{j}\left(\lambda^{\prime \prime} \Phi_{i}(t, x)\right)\right\}, \\
\vartheta_{c}(t):= & -f_{\mathbb{T}^{d}} \vartheta(t, x) d x .
\end{aligned}
$$

The factor $\left(D \Phi_{i}(t, x)\right)^{-1}$ is the inverse matrix of $D \Phi_{i}(t, x)$. Observe that for fixed $t_{0} \in[0,1]$, there are at most one odd index $i_{1}$ and one even index $i_{2}$ so that $\zeta_{i}(t)=0$ if $i \neq i_{1}, i_{2}$ and $t$ is close enough to $t_{0}$ (say, $\left|t-t_{0}\right| \leq 2 \tau / 3$ ). Therefore for such times $t$ we can write

$$
\begin{aligned}
\vartheta(t)= & \eta \psi(t)\left\{\zeta_{i_{1}}(t) \sum_{j=1}^{d} R_{0, j}(t) \Theta_{\mu^{\prime}}^{j}\left(\lambda^{\prime} \Phi_{i_{1}}(t)\right)\right. \\
& \left.+\zeta_{i_{2}}(t) \sum_{j=1}^{d} R_{0, j}(t) \Theta_{\mu^{\prime \prime}}^{j}\left(\lambda^{\prime \prime} \Phi_{i_{2}}(t)\right)\right\}, \\
w(t)= & \frac{\psi(t)}{\eta}\left\{\zeta_{i_{1}}(t) \sum_{j=1}^{d}\left(D \Phi_{i_{1}}(t)\right)^{-1} W_{\mu^{\prime}}^{j}\left(\lambda^{\prime} \Phi_{i_{1}}(t)\right)\right. \\
& \left.+\zeta_{i_{2}}(t) \sum_{j=1}^{d}\left(D \Phi_{i_{2}}(t)\right)^{-1} W_{\mu^{\prime \prime}}^{j}\left(\lambda^{\prime \prime} \Phi_{i_{2}}(t)\right)\right\},
\end{aligned}
$$


which we will call the fixed-time form of the perturbations. Notice that $\vartheta$ and $w$ are smooth functions. Notice also that $\vartheta+\vartheta_{c}$ has zero mean value in $\mathbb{T}^{d}$ at each time $t$. Finally observe that $w$ is a sum of terms of the form $(D \Phi)^{-1} G(\Phi)$, with

$$
\Phi=\Phi_{i}(t), \quad G=\left(W_{\mu^{\prime}}^{j}\right)_{\lambda^{\prime}} \text { or } G=\left(W_{\mu^{\prime \prime}}^{j}\right)_{\lambda^{\prime \prime}} .
$$

Since $\operatorname{div}\left(W_{\mu}\right)_{\lambda}=0$ for every $\mu, \lambda$ (see Proposition 5.1), we get from Lemma 3.2 that each one of these terms is divergence free and thus div $w=0$. Therefore

$$
\operatorname{div} u_{1}=\operatorname{div} u_{0}+\operatorname{div} w=0 .
$$

Remark 5.2 Observe that, thanks to the cutoff in time $\psi$, if $R_{0}(t) \equiv 0$, then

$$
\vartheta(t)=\vartheta_{c}(t)=w(t) \equiv 0
$$

\subsection{Estimates on the perturbation}

In this section we estimate $\vartheta, \vartheta_{c}, w$.

Lemma $5.3\left(L^{1}\right.$-norm of $\left.\vartheta\right)$. For every time $t \in[0,1]$,

$$
\|\vartheta(t)\|_{L^{1}\left(\mathbb{T}^{d}\right)} \leq \frac{M \eta}{2}\left\|R_{0}(t)\right\|_{L^{1}\left(\mathbb{T}^{d}\right)}+C\left(M, \eta,\left\|R_{0}\right\|_{C^{1}}, \max _{i=1, \ldots, N}\left\|D \Phi_{i}\right\|_{C^{0}}\right)\left(\frac{1}{\lambda^{\prime}}+\frac{1}{\lambda^{\prime \prime}}\right) .
$$

Proof. Since we have to estimate $\|\vartheta(t)\|_{L^{1}\left(\mathbb{T}^{d}\right)}$ for every fixed time $t$, we can assume that $\vartheta(t)$ has the form (56). In (56) each term in the summation over $j$ has the form $f \cdot\left(g_{\lambda} \circ \Phi\right)$, with

$$
\begin{aligned}
& f=R_{0, j}(t, \cdot) \quad f=R_{0, j}(t, \cdot), \\
& \Phi=\Phi_{i_{1}}(t, \cdot) \quad \Phi=\Phi_{i_{2}}(t, \cdot), \\
& g=\Theta_{\mu^{\prime}}^{j} \quad \text { or } \quad g=\Theta_{\mu^{\prime \prime}}^{j} \text {, } \\
& \lambda=\lambda^{\prime} \quad \lambda=\lambda^{\prime \prime} .
\end{aligned}
$$

Therefore we can apply the improved Hölder inequality, Lemma 3.4, to get

$$
\begin{aligned}
\|\vartheta(t)\|_{L^{1}} \leq & \eta\left\|R_{0}(t)\right\|_{L^{1}} \sum_{j=1}^{d}\left\|\Theta_{\mu^{\prime}}^{j}\right\|_{L^{1}} \\
& +\frac{C\left(\eta,\left\|R_{0}\right\|_{C^{1}}, \max _{i=1, \ldots, n}\left\|D \Phi_{i}\right\|_{C^{0}}\right)}{\lambda^{\prime}} \sum_{j=1}^{d}\left\|\Theta_{\mu^{\prime}}^{j}\right\|_{L^{1}} \\
& +\eta\left\|R_{0}(t)\right\|_{L^{1}} \sum_{j=1}^{d}\left\|\Theta_{\mu^{\prime \prime}}^{j}\right\|_{L^{1}} \\
& +\frac{C\left(\eta,\left\|R_{0}\right\|_{C^{1}}, \max _{i=1, \ldots, n}\left\|D \Phi_{i}\right\|_{C^{0}}\right)}{\lambda^{\prime \prime}} \sum_{j=1}^{d}\left\|\Theta_{\mu^{\prime \prime}}^{j}\right\|_{L^{1}} \\
\text { (by (50)) } \leq & \frac{M}{2} \eta\left\|R_{0}(t)\right\|_{L^{1}}+C\left(M, \eta,\left\|R_{0}\right\|_{C^{1}}, \max _{i=1, \ldots, n}\left\|D \Phi_{i}\right\|_{C^{0}}\right)\left(\frac{1}{\lambda^{\prime}}+\frac{1}{\lambda^{\prime \prime}}\right) .
\end{aligned}
$$


Lemma 5.4 (Estimate on $\vartheta_{c}$ ). For every time $t \in[0,1]$,

$$
\left|\vartheta_{C}(t)\right| \leq C\left(M, \eta,\left\|R_{0}\right\|_{C^{1}}, \max _{i=1, \ldots, N}\left\|D \Phi_{i}\right\|_{C^{0}}\right)\left(\frac{1}{\lambda^{\prime}}+\frac{1}{\lambda^{\prime \prime}}\right) .
$$

Proof. As in the proof of Lemma 5.3, we can use for $\vartheta(t)$ the form (56) and we observe that each term in the summation over $j$ has the form $f \cdot\left(g_{\lambda} \circ \Phi\right)$, with $f, \Phi, g, \lambda$ as in (57). We can thus apply Lemma 3.9 to get:

$$
\begin{aligned}
& \left|\vartheta_{c}(t)\right| \leq C\left(\eta,\left\|R_{0}\right\|_{C^{1}}, \max _{i=1, \ldots, n}\left\|D \Phi_{i}\right\|_{C^{0}}\right)\left[\frac{1}{\lambda^{\prime}} \sum_{j=1}^{d}\left\|\Theta_{\mu^{\prime}}^{j}\right\|_{L^{1}}+\frac{1}{\lambda^{\prime \prime}} \sum_{j=1}^{d}\left\|\Theta_{\mu^{\prime \prime}}^{j}\right\|_{L^{1}}\right] \\
& \text { (by }(50)) \leq C\left(M, \eta,\left\|R_{0}\right\|_{C^{1}}, \max _{i=1, \ldots, n}\left\|D \Phi_{i}\right\|_{C^{0}}\right)\left(\frac{1}{\lambda^{\prime}}+\frac{1}{\lambda^{\prime \prime}}\right) \text {. }
\end{aligned}
$$

Lemma $5.5\left(C^{0}\right.$ norm of $\left.w\right)$. For every time $t \in[0,1]$,

$$
\|w(t)\|_{C^{0}\left(\mathbb{T}^{d}\right)} \leq \frac{M}{2 \eta} \max _{i=1, \ldots, N}\left\|\left(D \Phi_{i}\right)^{-1}\right\|_{C^{0}\left(\operatorname{supp} \zeta_{i} \times \mathbb{T}^{d}\right)} .
$$

Proof. As in the proof of Lemma 5.3 we can use for $w(t)$ the form (56). Therefore

$$
\begin{aligned}
\|w(t)\|_{C^{0}\left(\mathbb{T}^{d}\right)} & \leq \frac{1}{\eta} \max _{i=1, \ldots, N}\left\|\left(D \Phi_{i}\right)^{-1}\right\|_{C^{0}\left(\operatorname{supp} \zeta_{i} \times \mathbb{T}^{d}\right)}\left(\sum_{j=1}^{d}\left\|W_{\mu^{\prime}}^{j}\right\|_{L^{\infty}\left(\mathbb{T}^{d}\right)}+\left\|W_{\mu^{\prime \prime}}^{j}\right\|_{L^{\infty}\left(\mathbb{T}^{d}\right)}\right) \\
(\operatorname{by}(50)) & \leq \frac{M}{2 \eta} \max _{i=1, \ldots, N}\left\|\left(D \Phi_{i}\right)^{-1}\right\|_{C^{0}\left(\operatorname{supp} \zeta_{i} \times \mathbb{T}^{d}\right)},
\end{aligned}
$$

which is what we wanted to prove.

Lemma $5.6\left(W^{1, p}\right.$ norm of $\left.w\right)$. For every time $t \in[0,1]$,

$$
\|w(t)\|_{W^{1, p}\left(\mathbb{T}^{d}\right)} \leq C\left(M, \eta, \max _{i=1, \ldots, N}\left\|D \Phi_{i}\right\|_{C^{1}}\right)\left(\lambda^{\prime}\left(\mu^{\prime}\right)^{1-(d-1) / p}+\lambda^{\prime \prime}\left(\mu^{\prime \prime}\right)^{1-(d-1) / p}\right) .
$$

Proof. As in the proof Lemma 5.5 we can use for $w(t)$ the form (56). Taking one partial derivative $\partial_{k}$, we get

$$
\begin{aligned}
\partial_{k} w(t)= & \frac{\psi(t)}{\eta}\left\{\zeta _ { i _ { 1 } } ( t ) \sum _ { j = 1 } ^ { d } \left[\partial_{k}\left(D \Phi_{i_{1}}(t)\right)^{-1} W_{\mu^{\prime}}^{j}\left(\lambda^{\prime} \Phi_{i_{1}}(t)\right)\right.\right. \\
& \left.+\lambda^{\prime}\left(D \Phi_{i_{1}}(t)\right)^{-1} D W_{\mu^{\prime}}^{j}\left(\lambda^{\prime} \Phi_{i_{1}}(t)\right) D \Phi_{i_{1}}(t) e_{k}\right] \\
& +\zeta_{i_{2}}(t) \sum_{j=1}^{d}\left[\partial_{k}\left(D \Phi_{i_{2}}(t)\right)^{-1} W_{\mu^{\prime \prime}}^{j}\left(\lambda^{\prime \prime} \Phi_{i_{2}}(t)\right)\right. \\
& \left.\left.+\lambda^{\prime \prime}\left(D \Phi_{i_{2}}(t)\right)^{-1} D W_{\mu^{\prime \prime}}^{j}\left(\lambda^{\prime \prime} \Phi_{i_{2}}(t)\right) D \Phi_{i_{2}}(t) e_{k}\right]\right\} .
\end{aligned}
$$


We now apply the classical Hölder inequality to estimate $\left\|\partial_{k} w(t)\right\|_{L^{p}}$ :

$$
\begin{aligned}
& \left\|\partial_{k} w(t)\right\|_{L^{p}} \leq \frac{\psi(t)}{\eta}\left\{\max _{i=1, \ldots, N}\left\|D\left(D \Phi_{i}\right)^{-1}\right\|_{C^{0}}\left(\sum_{j=1}^{d}\left\|W_{\mu^{\prime}}^{j}\right\|_{L^{p}}+\left\|W_{\mu^{\prime \prime}}^{j}\right\|_{L^{p}}\right)\right. \\
& \left.+\max _{i=1, \ldots, N}\left\|\left(D \Phi_{i}\right)^{-1}\right\|_{C^{0}}\left\|D \Phi_{i}\right\|_{C^{0}} \cdot \cdot\left(\lambda^{\prime} \sum_{j=1}^{d}\left\|D W_{\mu^{\prime}}^{j}\right\|_{L^{p}}+\lambda^{\prime \prime} \sum_{j=1}^{d}\left\|D W_{\mu^{\prime \prime}}^{j}\right\|_{L^{p}}\right)\right\} \\
& \text { (by Lemma 3.1) } \leq C\left(\eta, \max _{i=1, \ldots, N}\left\|D \Phi_{i}\right\|_{C^{1}}\right)\left(\lambda^{\prime} \sum_{j=1}^{d}\left\|W_{\mu^{\prime}}^{j}\right\|_{W^{1, p}}+\lambda^{\prime \prime} \sum_{j=1}^{d}\left\|W_{\mu^{\prime \prime}}^{j}\right\|_{W^{1, p}}\right) \\
& \text { (by }(51)) \leq C\left(M, \eta, \max _{i=1, \ldots, N}\left\|D \Phi_{i}\right\|_{C^{1}}\right)\left(\lambda^{\prime}\left(\mu^{\prime}\right)^{1-(d-1) / p}+\lambda^{\prime \prime}\left(\mu^{\prime \prime}\right)^{1-(d-1) / p}\right) .
\end{aligned}
$$

A similar (and even easier) computation holds for $\|w(t)\|_{L^{p}}$, thus concluding the proof of the lemma.

\section{The new defect field}

In this section we continue the proof of Proposition 4.1, defining the new defect field $R_{1}$ and estimating it.

\subsection{Definition of the new defect field}

We want to define $R_{1}$ so that

$$
-\operatorname{div} R_{1}=\partial_{t} \rho_{1}+\operatorname{div}\left(\rho_{1} u_{1}\right) .
$$

Let us compute

$$
\begin{aligned}
& \partial_{t} \rho_{1}+\operatorname{div}\left(\rho_{1} u_{1}\right)=\operatorname{div}\left(\vartheta w-R_{0}\right) \\
& \quad+\left[\partial_{t}\left(\vartheta+\vartheta_{c}\right)+\operatorname{div}\left(\left(\vartheta+\vartheta_{c}\right) u_{0}\right)\right] \\
& \quad+\operatorname{div}\left(\rho_{0} w\right)+\operatorname{div}\left(\vartheta_{c} w\right) \\
& =\operatorname{div}\left(R^{\text {interaction }}+R^{\text {flow }}+R^{\psi}+R^{\text {quadr }}\right) \\
& \quad+\operatorname{div} R^{\text {transport }} \\
& \quad+\operatorname{div} R^{\text {Nash }}+\operatorname{div} R^{\text {corr }}
\end{aligned}
$$

where we put

$$
R^{\mathrm{Nash}}:=\rho_{0} w, \quad R^{\mathrm{corr}}:=\vartheta_{c} w
$$

and $R^{\text {interaction }}, R^{\text {flow }}, R^{\psi}, R^{\text {quadr }}, R^{\text {transport }}$ will be defined respectively in (63), (64), (65), (66), (70) in such a way that

$$
\begin{aligned}
& \operatorname{div}\left(\vartheta w-R_{0}\right)=\operatorname{div}\left(R^{\text {interaction }}+R^{\text {flow }}+R^{\psi}+R^{\text {quadr }}\right), \\
& \partial_{t}\left(\vartheta+\vartheta_{c}\right)+\operatorname{div}\left(\left(\vartheta+\vartheta_{c}\right) u_{0}\right)=\operatorname{div} R^{\text {transport }} .
\end{aligned}
$$

We thus define

$$
-R_{1}:=R^{\text {interaction }}+R^{\text {flow }}+R^{\psi}+R^{\text {quadr }}+R^{\text {transport }}+R^{\text {Nash }}+R^{\text {corr }},
$$

so that (58) holds. 


\subsection{Definition and estimates for $R^{\text {interaction }}, R^{\text {flow }}, R^{\Psi}, R^{\text {quadr }}$}

In this section we define and estimate the vector fields $R^{\text {quadr }}, R^{\text {interaction }}, R^{\psi}$ and $R^{\text {flow }}$ so that (61a) holds. First of all, we want to compute more explicitly div $\left(\vartheta(t) w(t)-R_{0}(t)\right)$, for every fixed time $t$. We can use the form (56) for $\vartheta(t)$ and $w(t)$. Exploiting the fact that for $j \neq k, \Theta_{\mu}^{j}$ and $W_{\mu}^{k}$ have disjoint support (see Proposition 5.1), we have

$$
\begin{aligned}
\vartheta(t) w(t)= & \psi^{2}(t)\left\{\zeta_{i_{1}}^{2}(t) \sum_{j=1}^{d} R_{0, j}(t)\left(D \Phi_{i_{1}}(t)\right)^{-1} \Theta_{\mu^{\prime}}^{j}\left(\lambda^{\prime} \Phi_{i_{1}}(t)\right) W_{\mu^{\prime}}^{j}\left(\lambda^{\prime} \Phi_{i_{1}}(t)\right)\right. \\
& \left.+\zeta_{i_{2}}^{2}(t) \sum_{j=1}^{d} R_{0, j}(t)\left(D \Phi_{i_{2}}(t)\right)^{-1} \Theta_{\mu^{\prime \prime}}^{j}\left(\lambda^{\prime \prime} \Phi_{i_{2}}(t)\right) W_{\mu^{\prime \prime}}^{j}\left(\lambda^{\prime \prime} \Phi_{i_{2}}(t)\right)\right\} \\
& +R^{\text {interaction }}(t),
\end{aligned}
$$

where we set

$$
\begin{aligned}
& R^{\text {interaction }}(t):=\psi^{2}(t) \zeta_{i_{1}}(t) \zeta_{i_{2}}(t) \\
& \sum_{j, k=1}^{d}\left[R_{0, j}(t)\left(D \Phi_{i_{2}}(t)\right)^{-1} \Theta_{\mu^{\prime}}^{j}\left(\lambda^{\prime} \Phi_{i_{1}}(t)\right) W_{\mu^{\prime \prime}}^{k}\left(\lambda^{\prime \prime} \Phi_{i_{2}}(t)\right)\right. \\
& \left.\quad+R_{0, j}(t)\left(D \Phi_{i_{1}}(t)\right)^{-1} \Theta_{\mu^{\prime \prime}}^{j}\left(\lambda^{\prime \prime} \Phi_{i_{2}}(t)\right) W_{\mu^{\prime}}^{k}\left(\lambda^{\prime} \Phi_{i_{1}}(t)\right)\right] .
\end{aligned}
$$

On the other side, using the fact that $\sum_{i=1}^{N} \zeta_{i}^{2} \equiv 1$, we can write

$$
\begin{aligned}
R_{0}(t)= & \psi^{2}(t) R_{0}(t)+R_{0}(t)\left[1-\psi^{2}(t)\right] \\
= & \psi^{2}(t) R_{0}(t)-R^{\psi}(t) \\
& \text { (using that } \left.\sum_{i=1}^{N} \zeta_{i}^{2} \equiv 1\right) \\
= & \psi^{2}(t)\left\{\zeta_{i_{1}}^{2}(t) R_{0}(t)+\zeta_{i_{2}}^{2}(t) R_{0}(t)\right\}-R^{\psi}(t) \\
= & \psi^{2}(t)\left\{\zeta_{i_{1}}^{2}(t)\left[D \Phi_{i_{1}}(t)\right]^{-1} R_{0}(t)+\zeta_{i_{2}}^{2}(t)\left[D \Phi_{i_{2}}(t)\right]^{-1} R_{0}(t)\right\}-R^{\text {flow }}(t)-R^{\psi}(t) \\
= & \psi^{2}(t)\left\{\zeta_{i_{1}}^{2}(t) \sum_{j=1}^{d} R_{0, j}(t)\left[D \Phi_{i_{1}}(t)\right]^{-1} e_{j}+\zeta_{i_{2}}^{2}(t) \sum_{j=1}^{d} R_{0, j}(t)\left[D \Phi_{i_{2}}(t)\right]^{-1} e_{j}\right\} \\
& \quad-R^{\text {flow }}(t)-R^{\psi}(t),
\end{aligned}
$$

where we set

$$
\begin{aligned}
-R^{\text {flow }}(t):= & \psi^{2}(t)\left\{\zeta_{i_{1}}^{2}(t)\left[\operatorname{Id}-\left(D \Phi_{i_{1}}(t)\right)^{-1}\right] R_{0}(t)\right. \\
& \left.+\zeta_{i_{2}}^{2}(t)\left[\operatorname{Id}-\left(D \Phi_{i_{2}}(t)\right)^{-1}\right] R_{0}(t)\right\}
\end{aligned}
$$

with Id being the identity matrix, and

$$
-R^{\psi}(t):=R_{0}(t)\left[1-\psi^{2}(t)\right] .
$$


Summarizing, we have

$$
\begin{aligned}
\operatorname{div}( & \left.\vartheta(t) w(t)-R_{0}(t)\right) \\
= & \operatorname{div} R^{\text {interaction }}(t)+\operatorname{div} R^{\text {flow }}(t)+\operatorname{div} R^{\psi}(t) \\
& +\psi^{2}(t)\left\{\zeta_{i_{1}}^{2}(t) \sum_{j=1}^{d} \operatorname{div}\left[R_{0, j}\left[D \Phi_{i_{1}}(t)\right]^{-1}\left(\Theta_{\mu^{\prime}}^{j}\left(\lambda^{\prime} \Phi_{i_{1}}(t)\right) W_{\mu^{\prime}}^{j}\left(\lambda^{\prime} \Phi_{i_{1}}(t)\right)-e_{j}\right)\right]\right. \\
& \left.+\zeta_{i_{2}}^{2}(t) \sum_{j=1}^{d} \operatorname{div}\left[R_{0, j}\left[D \Phi_{i_{2}}(t)\right]^{-1}\left(\Theta_{\mu^{\prime \prime}}^{j}\left(\lambda^{\prime \prime} \Phi_{i_{2}}(t)\right) W_{\mu^{\prime \prime}}^{j}\left(\lambda^{\prime \prime} \Phi_{i_{2}}(t)\right)-e_{j}\right)\right]\right\} \\
= & \operatorname{div} R^{\text {interaction }}(t)+\operatorname{div} R^{\text {flow }}(t)+\operatorname{div} R^{\psi}(t) \\
& +\psi^{2}(t)\left\{\zeta_{i_{1}}^{2}(t) \sum_{j=1}^{d} \nabla R_{0, j} \cdot\left[D \Phi_{i_{1}}(t)\right]^{-1}\left(\Theta_{\mu^{\prime}}^{j}\left(\lambda^{\prime} \Phi_{i_{1}}(t)\right) W_{\mu^{\prime}}^{j}\left(\lambda^{\prime} \Phi_{i_{1}}(t)\right)-e_{j}\right)\right. \\
& \left.+\zeta_{i_{2}}^{2}(t) \sum_{j=1}^{d} \nabla R_{0, j} \cdot\left[D \Phi_{i_{2}}(t)\right]^{-1}\left(\Theta_{\mu^{\prime \prime}}^{j}\left(\lambda^{\prime \prime} \Phi_{i_{2}}(t)\right) W_{\mu^{\prime \prime}}^{j}\left(\lambda^{\prime \prime} \Phi_{i_{2}}(t)\right)-e_{j}\right)\right\},
\end{aligned}
$$

where in the last equality we used the fact that $\operatorname{div}\left(\left(\Theta_{\mu}^{j}\right)_{\lambda}\left(W_{\mu}^{j}\right)_{\lambda}-e_{j}\right)=0$ for every $\mu, \lambda, j$ (see Proposition 5.1) and Lemma 3.2. We now observe that each term in the two summations over $j$ has zero mean value (being a divergence) and it has the form $f(D \Phi)^{-1}\left(g_{\lambda} \circ \Phi\right)$, for

$$
\begin{aligned}
& f=\nabla R_{0, j}(t, \cdot) \quad f=\nabla R_{0, j}(t, \cdot), \\
& \Phi=\Phi_{i_{1}}(t, \cdot) \quad \Phi=\Phi_{i_{2}}(t, \cdot), \\
& g=\Theta_{\mu^{\prime}}^{j} W_{\mu^{\prime}}^{j}-e_{j} \text { or } \quad g=\Theta_{\mu^{\prime \prime}}^{j} W_{\mu^{\prime \prime}}^{j}-e_{j} \text {, } \\
& \lambda=\lambda^{\prime} \quad \lambda=\lambda^{\prime \prime} .
\end{aligned}
$$

We can therefore apply Lemma 3.6 and define

$$
\begin{aligned}
& R^{\text {quadr }}(t) \\
& :=\psi^{2}(t)\left\{\zeta_{i_{1}}^{2}(t) \sum_{j=1}^{d} \mathcal{R}\left(\nabla R_{0, j} \cdot\left[D \Phi_{i_{1}}(t)\right]^{-1}\left(\Theta_{\mu^{\prime}}^{j}\left(\lambda^{\prime} \Phi_{i_{1}}(t)\right) W_{\mu^{\prime}}^{j}\left(\lambda^{\prime} \Phi_{i_{1}}(t)\right)-e_{j}\right)\right)\right. \\
& \left.\quad+\zeta_{i_{2}}^{2}(t) \sum_{j=1}^{d} \mathcal{R}\left(\nabla R_{0, j} \cdot\left[D \Phi_{i_{2}}(t)\right]^{-1}\left(\Theta_{\mu^{\prime \prime}}^{j}\left(\lambda^{\prime \prime} \Phi_{i_{2}}(t)\right) W_{\mu^{\prime \prime}}^{j}\left(\lambda^{\prime \prime} \Phi_{i_{2}}(t)\right)-e_{j}\right)\right)\right\},
\end{aligned}
$$

so that (61a) holds. We now separately estimate $R^{\text {interaction }}, R^{\text {flow }}, R^{\psi}, R^{\text {quadr }}$. We start with $R^{\text {interaction }}$.

\section{Lemma 6.1 For every time $t$ it holds}

$$
\begin{aligned}
\left\|R^{\text {interaction }}(t)\right\|_{L^{1}\left(\mathbb{T}^{d}\right)} & \leq C\left(M,\left\|R_{0}\right\|_{\left.C^{0}, \max _{i=1, \ldots, N}\left\|D \Phi_{i}\right\|_{C^{0}}\right)}\right. \\
& \left(\frac{1}{\left(\mu^{\prime}\right)^{d-1}}+\frac{1}{\left(\mu^{\prime \prime}\right)^{d-1}}+\frac{\lambda^{\prime} \mu^{\prime}}{\lambda^{\prime \prime}}+\frac{\lambda^{\prime}\left(\mu^{\prime}\right)^{d}}{\lambda^{\prime \prime}\left(\mu^{\prime \prime}\right)^{d-1}}\right) .
\end{aligned}
$$


Proof. Consider the definition (63) of $R^{\text {interaction }}$. We start by estimating $\left\|\Theta_{\mu^{\prime}}^{j}\left(\lambda^{\prime} \Phi_{i_{1}}(t)\right)\right\|_{C^{1}}$ and $\left\|W_{\mu^{\prime}}^{k}\left(\lambda^{\prime} \Phi_{i_{1}}(t)\right)\right\|_{C^{1}}$, using (52) and the chain rule

$$
\begin{aligned}
\left\|\Theta_{\mu^{\prime}}^{j}\left(\lambda^{\prime} \Phi_{i_{1}}(t)\right)\right\|_{C^{1}} & \leq C\left(M, \max _{i=1, \ldots, N}\left\|D \Phi_{i}\right\|_{C^{0}}\right) \lambda^{\prime}\left(\mu^{\prime}\right)^{d}, \\
\left\|W_{\mu^{\prime}}^{k}\left(\lambda^{\prime} \Phi_{i_{1}}(t)\right)\right\|_{C^{1}} & \leq C\left(M, \max _{i=1, \ldots, N}\left\|D \Phi_{i}\right\|_{C^{0}}\right) \lambda^{\prime} \mu^{\prime} .
\end{aligned}
$$

We now estimate $\Theta_{\mu^{\prime}}^{j}\left(\lambda^{\prime} \Phi_{i_{1}}(t)\right) W_{\mu^{\prime \prime}}^{k}\left(\lambda^{\prime \prime} \Phi_{i_{2}}(t)\right)$, using the improved Hölder inequality, Lemma 3.4 and considering $\lambda^{\prime \prime}$ as the fast oscillation. We have

$$
\begin{aligned}
& \left\|\Theta_{\mu^{\prime}}^{j}\left(\lambda^{\prime} \Phi_{i_{1}}(t)\right) W_{\mu^{\prime \prime}}^{k}\left(\lambda^{\prime \prime} \Phi_{i_{2}}(t)\right)\right\|_{L^{1}} \\
& \quad \leq\left\|\Theta_{\mu^{\prime}}^{j}\right\|_{L^{1}}\left\|W_{\mu^{\prime \prime}}^{k}\right\|_{L^{1}}+\frac{1}{\lambda^{\prime \prime}}\left\|\Theta_{\mu^{\prime}}^{j}\left(\lambda^{\prime} \Phi_{i_{1}}(t)\right)\right\|_{C^{1}}\left\|D \Phi_{i_{2}}(t)\right\|_{C^{0}}^{d-1}\left\|W_{\mu^{\prime \prime}}^{k}\right\|_{L^{1}} \\
& \quad \leq C\left(M, \max _{i=1, \ldots, N}\left\|D \Phi_{i}\right\|_{C^{0}}\right)\left(\frac{1}{\left(\mu^{\prime \prime}\right)^{d-1}}+\frac{\lambda^{\prime}\left(\mu^{\prime}\right)^{d}}{\lambda^{\prime \prime}\left(\mu^{\prime \prime}\right)^{d-1}}\right),
\end{aligned}
$$

where in the last inequality we used (50), (51) and (67). A similar estimate holds for $\Theta_{\mu^{\prime \prime}}^{j}\left(\lambda^{\prime \prime} \Phi_{i_{2}}(t)\right) W_{\mu^{\prime}}^{k}\left(\lambda^{\prime} \Phi_{i_{1}}(t)\right)$ :

$$
\begin{aligned}
& \left\|\Theta_{\mu^{\prime \prime}}^{j}\left(\lambda^{\prime \prime} \Phi_{i_{2}}(t)\right) W_{\mu^{\prime}}^{k}\left(\lambda^{\prime} \Phi_{i_{1}}(t)\right)\right\|_{L^{1}} \\
& \quad \leq\left\|\Theta_{\mu^{\prime \prime}}^{j}\right\|_{L^{1}}\left\|W_{\mu^{\prime}}^{k}\right\|_{L^{1}}+\frac{1}{\lambda^{\prime \prime}}\left\|W_{\mu^{\prime}}^{k}\left(\lambda^{\prime} \Phi_{i_{1}}(t)\right)\right\|_{C^{1}}\left\|D \Phi_{i_{2}}(t)\right\|_{C^{0}}^{d-1}\left\|\Theta_{\mu^{\prime \prime}}^{j}\right\|_{L^{1}} \\
& \quad \leq C\left(M, \max _{i=1, \ldots, N}\left\|D \Phi_{i}\right\|_{C^{0}}\right)\left(\frac{1}{\left(\mu^{\prime}\right)^{d-1}}+\frac{\lambda^{\prime} \mu^{\prime}}{\lambda^{\prime \prime}}\right) .
\end{aligned}
$$

Therefore

$$
\begin{aligned}
\| & R^{\text {interaction }}(t) \|_{L^{1}} \\
\leq & C\left(M,\left\|R_{0}\right\|_{\left.C^{0}, \max _{i=1, \ldots, N}\left\|D \Phi_{i}\right\|_{C^{0}}\right) .}\right. \\
& \cdot \sum_{j, k=1}^{d}\left[\left\|\Theta_{\mu^{\prime}}^{j}\left(\lambda^{\prime} \Phi_{i_{1}}(t)\right) W_{\mu^{\prime \prime}}^{k}\left(\lambda^{\prime \prime} \Phi_{i_{2}}(t)\right)\right\|_{L^{1}}+\left\|\Theta_{\mu^{\prime \prime}}^{j}\left(\lambda^{\prime \prime} \Phi_{i_{2}}(t)\right) W_{\mu^{\prime}}^{k}\left(\lambda^{\prime} \Phi_{i_{1}}(t)\right)\right\|_{L^{1}}\right] \\
\leq & C\left(M,\left\|R_{0}\right\|_{C^{0},} \max _{i=1, \ldots, N}\left\|D \Phi_{i}\right\|_{C^{0}}\right)\left(\frac{1}{\left(\mu^{\prime}\right)^{d-1}}+\frac{1}{\left(\mu^{\prime \prime}\right)^{d-1}}+\frac{\lambda^{\prime} \mu^{\prime}}{\lambda^{\prime \prime}}+\frac{\lambda^{\prime}\left(\mu^{\prime}\right)^{d}}{\lambda^{\prime \prime}\left(\mu^{\prime \prime}\right)^{d-1}}\right) .
\end{aligned}
$$

Lemma 6.2 For every $t \in[0,1]$,

$$
\left\|R^{\text {flow }}(t)\right\|_{L^{1}\left(\mathbb{T}^{d}\right)} \leq\left\|R_{0}\right\|_{C^{0}} \max _{i=1, \ldots, N}\left\|\operatorname{Id}-D \Phi_{i}(t)^{-1}\right\|_{C^{0}\left(\operatorname{supp} \zeta_{i} \times \mathbb{T}^{d}\right)} .
$$

Proof. The proof follows immediately from the definition of $R^{\text {flow }}$.

Lemma 6.3 For every $t \in[0,1]$,

$$
\left\|R^{\psi}(t)\right\|_{L^{1}\left(\mathbb{T}^{d}\right)} \leq \delta / 4 .
$$

Proof. If $\psi^{2}(t) \neq 1$, then, by (54), $\left\|R_{0}(t)\right\|_{L^{1}} \leq \delta / 4$ and thus the conclusion follows.

Lemma 6.4 For every $t \in[0,1]$,

$$
\left\|R^{\text {quadr }}(t)\right\|_{L^{1}\left(\mathbb{T}^{d}\right)} \leq C\left(M,\left\|R_{0}\right\|_{C^{2},} \max _{i=1, \ldots, N}\left\|D \Phi_{i}\right\|_{C^{1}}\right)\left(\frac{1}{\lambda^{\prime}}+\frac{1}{\lambda^{\prime \prime}}\right) .
$$


Proof. $R^{\text {quadr }}(t)$ is defined in (66) using Lemma 3.6. Applying the bounds provided by such proposition, with $k=0$ and $p=1$, we get

$$
\begin{aligned}
\left\|R^{\text {quadr }}(t)\right\|_{L^{1}} \leq & C\left(\left\|R_{0}\right\|_{C^{2}}, \max _{i=1, \ldots, N}\left\|D \Phi_{i}\right\|_{C^{1}}\right) \\
& \left(\frac{1}{\lambda^{\prime}} \sum_{j=1}^{d}\left\|\Theta_{\mu^{\prime}}^{j} W_{\mu^{\prime}}^{j}-e_{j}\right\|_{L^{1}}+\frac{1}{\lambda^{\prime \prime}} \sum_{j=1}^{d}\left\|\Theta_{\mu^{\prime \prime}}^{j} W_{\mu^{\prime \prime}}^{j}-e_{j}\right\|_{L^{1}}\right)(\text { by (50)) } \\
\leq & C\left(M,\left\|R_{0}\right\|_{C^{2},} \max _{i=1, \ldots, N}\left\|D \Phi_{i}\right\|_{C^{1}}\right)\left(\frac{1}{\lambda^{\prime}}+\frac{1}{\lambda^{\prime \prime}}\right)
\end{aligned}
$$

thus concluding the proof of the lemma.

\subsection{Definition and estimates for $R^{\text {transport }}$}

In this section we define and estimate the vector fields $R^{\text {transport }}$ so that (61b) holds. First of all, we want to compute more explicitly $\partial_{t}\left(\vartheta(t)+\vartheta_{c}(t)\right)+\operatorname{div}\left(\left(\vartheta(t)+\vartheta_{c}(t)\right) u_{0}(t)\right)$, for every fixed time $t$. We can use the fixed-time form (56) for $\vartheta(t)$ and $w(t)$. We have

$$
\begin{aligned}
\partial_{t}(\vartheta & \left.+\vartheta_{c}\right)+\operatorname{div}\left(\left(\vartheta+\vartheta_{c}\right) u_{0}\right) \\
= & \dot{\vartheta}_{c}+\sum_{j=1}^{d}\left\{A_{1}^{j} \Theta_{\mu^{\prime}}^{j}\left(\lambda^{\prime} \Phi_{i_{1}}\right)+A_{2}^{j} \Theta_{\mu^{\prime \prime}}^{j}\left(\lambda^{\prime \prime} \Phi_{i_{2}}\right)\right. \\
& \left.+\lambda^{\prime} B_{1}^{j} \cdot\left[\partial_{t} \Phi_{i_{1}}+\left(u_{0} \cdot \nabla\right) \Phi_{i_{1}}\right]+\lambda^{\prime \prime} B_{2}^{j} \cdot\left[\partial_{t} \Phi_{i_{2}}+\left(u_{0} \cdot \nabla\right) \Phi_{i_{2}}\right]\right\} \\
= & \dot{\vartheta}_{c}+\sum_{j=1}^{d}\left\{A_{1}^{j} \Theta_{\mu^{\prime}}^{j}\left(\lambda^{\prime} \Phi_{i_{1}}\right)+A_{2}^{j} \Theta_{\mu^{\prime \prime}}^{j}\left(\lambda^{\prime \prime} \Phi_{i_{2}}\right)\right\},
\end{aligned}
$$

where

$$
\begin{aligned}
A_{1}^{j} & :=\eta\left[\dot{\psi} \zeta_{i_{1}} R_{0, j}+\psi \dot{\zeta}_{i_{1}} R_{0, j}+\psi \zeta_{i_{1}}\left(\partial_{t} R_{0, j}+\nabla R_{0, j} \cdot u_{0}\right)\right], \\
A_{2}^{j} & :=\eta\left[\dot{\psi} \zeta_{i_{2}} R_{0, j}+\psi \dot{\zeta}_{i_{2}} R_{0, j}+\psi \zeta_{i_{2}}\left(\partial_{t} R_{0, j}+\nabla R_{0, j} \cdot u_{0}\right)\right], \\
B_{1}^{j} & :=\eta \psi \zeta_{i_{1}} R_{0, j} \nabla \Theta_{\mu^{\prime}}^{j}\left(\lambda^{\prime} \Phi_{i_{1}}\right), \\
B_{2}^{j} & :=\eta \psi \zeta_{i_{2}} R_{0, j} \nabla \Theta_{\mu^{\prime \prime}}^{j}\left(\lambda^{\prime \prime} \Phi_{i_{2}}\right),
\end{aligned}
$$

and we used (53). Here we used the notation $\dot{f}=d f / d t$, if $f=f(t)$ is a function depending only on time. We now continue the chain of equalities in (68), by adding and subtracting the mean value of each term in the summations over $j$, as follows:

$$
\begin{aligned}
\partial_{t}(\vartheta & \left.+\vartheta_{c}\right)+\operatorname{div}\left(\left(\vartheta+\vartheta_{c}\right) u_{0}\right) \\
= & \sum_{j=1}^{d}\left\{\left(A_{1}^{j} \Theta_{\mu^{\prime}}^{j}\left(\lambda^{\prime} \Phi_{i_{1}}\right)-f_{T^{d}} A_{1}^{j} \Theta_{\mu^{\prime}}^{j}\left(\lambda^{\prime} \Phi_{i_{1}}\right) d x\right)\right. \\
& \left.+\left(A_{2}^{j} \Theta_{\mu^{\prime \prime}}^{j}\left(\lambda^{\prime \prime} \Phi_{i_{2}}\right)-f_{T^{d}} A_{2}^{j} \Theta_{\mu^{\prime \prime}}^{j}\left(\lambda^{\prime \prime} \Phi_{i_{2}}\right) d x\right)\right\}
\end{aligned}
$$




$$
\begin{aligned}
& +\dot{\vartheta}_{c}+\sum_{j=1}^{d}\left\{f_{T^{d}} A_{1}^{j} \Theta_{\mu^{\prime}}^{j}\left(\lambda^{\prime} \Phi_{i_{1}}\right) d x+f_{T^{d}} A_{2}^{j} \Theta_{\mu^{\prime \prime}}^{j}\left(\lambda^{\prime \prime} \Phi_{i_{2}}\right) d x\right\} \\
& =\sum_{j=1}^{d}\left\{\left(A_{1}^{j} \Theta_{\mu^{\prime}}^{j}\left(\lambda^{\prime} \Phi_{i_{1}}\right)-f_{T^{d}} A_{1}^{j} \Theta_{\mu^{\prime}}^{j}\left(\lambda^{\prime} \Phi_{i_{1}}\right) d x\right)\right. \\
& \left.+\left(A_{2}^{j} \Theta_{\mu^{\prime \prime}}^{j}\left(\lambda^{\prime \prime} \Phi_{i_{2}}\right)-f_{T^{d}} A_{2}^{j} \Theta_{\mu^{\prime \prime}}^{j}\left(\lambda^{\prime \prime} \Phi_{i_{2}}\right) d x\right)\right\} .
\end{aligned}
$$

The last equality is a consequence of the fact that

$$
f\left(\partial_{t}\left(\vartheta+\vartheta_{c}\right)+\operatorname{div}\left(\left(\vartheta+\vartheta_{c}\right) u_{0}\right)\right) d x=0 .
$$

We now observe that, at the fixed time $t$, each term in the last line in (69) has the form $f \cdot\left(g_{\lambda} \circ \Phi\right)-f f \cdot\left(g_{\lambda} \circ \Phi\right) d x$ for

$$
\begin{array}{lll}
f=A_{1}^{j}(t, \cdot) & \text { or } & f=A_{2}^{j}(t, \cdot), \\
\Phi=\Phi_{i_{1}}(t, \cdot) & \text { or } & \Phi=\Phi_{i_{2}}(t, \cdot), \\
g=\Theta_{\mu^{\prime}}^{j} & \text { or } & g=\Theta_{\mu^{\prime \prime}}^{j}, \\
\lambda=\lambda^{\prime} & \text { or } & \lambda=\lambda^{\prime \prime} .
\end{array}
$$

Since $\Theta_{\mu}^{j}$ has zero mean value (see Proposition 5.1), we can apply Lemma 3.6 and define

$$
\begin{aligned}
R^{\text {transport }}(t):= & \sum_{j=1}^{d}\left\{\mathcal{R}\left(A_{1}^{j}(t, \cdot) \Theta_{\mu^{\prime}}^{j}\left(\lambda^{\prime} \Phi_{i_{1}}\right)-f_{T^{d}} A_{1}^{j}(t, \cdot) \Theta_{\mu^{\prime}}^{j}\left(\lambda^{\prime} \Phi_{i_{1}}\right) d x\right)\right. \\
& \left.+\mathcal{R}\left(A_{2}^{j}(t, \cdot) \Theta_{\mu^{\prime \prime}}^{j}\left(\lambda^{\prime \prime} \Phi_{i_{2}}\right)-f_{T^{d}} A_{2}^{j}(t, \cdot) \Theta_{\mu^{\prime \prime}}^{j}\left(\lambda^{\prime \prime} \Phi_{i_{2}}\right) d x\right)\right\} .
\end{aligned}
$$

Lemma 6.5 For every $t \in[0,1]$, it holds

$$
\left\|R^{\text {transport }}(t)\right\|_{L^{1}\left(\mathbb{T}^{d}\right)} \leq C\left(M, \eta, \delta, \tau,\left\|R_{0}\right\|_{C^{2}},\left\|u_{0}\right\|_{C^{1}}, \max _{i=1, \ldots, N}\left\|D \Phi_{i}\right\|_{C^{1}}\right)\left(\frac{1}{\lambda^{\prime}}+\frac{1}{\lambda^{\prime \prime}}\right) .
$$

Proof. First of all, we observe that

$$
\left|\psi^{\prime}(t)\right| \leq C\left(\delta,\left\|R_{0}\right\|_{C^{1}}\right), \quad\left|\zeta_{i}^{\prime}(t)\right| \leq C(\tau) \text { for all } i=1, \ldots, N .
$$

Therefore

$$
\left\|A_{1}^{j}(t)\right\|_{C^{1}},\left\|A_{2}^{j}(t)\right\|_{C^{1}} \leq C\left(\eta, \delta, \tau,\left\|R_{0}\right\|_{C^{2}},\left\|u_{0}\right\|_{C^{1}}\right) .
$$

We defined $R^{\text {transport }}$ in (70) using the antidivergence operator provided by Lemma 3.6. We can thus apply the bounds provided by such proposition, with $k=0$ and $p=1$, to get

$$
\begin{aligned}
& \left\|R^{\text {transport }}(t)\right\|_{L^{1}} \\
& \leq \sum_{j=1}^{d}\left\{\frac{\left\|A_{1}^{j}(t)\right\|_{C^{1}}\left\|D \Phi_{i_{1}}\right\|_{C^{1}}^{d-1}\left\|\Theta_{\mu^{\prime}}^{j}\right\|_{L^{1}}}{\lambda^{\prime}}+\frac{\left\|A_{2}^{j}(t)\right\|_{C^{1}}\left\|D \Phi_{i_{2}}\right\|_{C^{1}}^{d-1}\left\|\Theta_{\mu^{\prime \prime}}^{j}\right\|_{L^{1}}}{\lambda^{\prime \prime}}\right\} \\
& \leq C\left(M, \eta, \delta, \tau,\left\|R_{0}\right\|_{C^{2}},\left\|u_{0}\right\|_{C^{1}}, \max _{i=1, \ldots, N}\left\|D \Phi_{i}\right\|_{C^{1}}\right)\left(\frac{1}{\lambda^{\prime}}+\frac{1}{\lambda^{\prime \prime}}\right),
\end{aligned}
$$

where in the last line we used (50). 


\subsection{Estimates for $R^{\text {Nash }}$ and $R^{\text {corr }}$}

In this section we estimate $R^{\mathrm{Nash}}$ and $R^{\text {corr }}$.

Lemma 6.6 For every $t \in[0,1]$,

$$
\left\|R^{\mathrm{Nash}}(t)\right\|_{L^{1}\left(\mathbb{T}^{d}\right)} \leq C\left(\left\|\rho_{0}(t)\right\|_{C^{0},} \max _{i=1, \ldots, N}\left\|D \Phi_{i}\right\|_{C^{0}}\right)\left(\frac{1}{\left(\mu^{\prime}\right)^{d-1}}+\frac{1}{\left(\mu^{\prime \prime}\right)^{d-1}}\right) .
$$

Proof. We have

$$
\begin{aligned}
\left\|R^{\mathrm{Nash}}(t)\right\|_{L^{1}} & =\left\|\rho_{0}(t) w(t)\right\|_{L^{1}} \\
& \leq\left\|\rho_{0}(t)\right\|_{C^{0}}\|w(t)\|_{L^{1}}
\end{aligned}
$$

(by Lemma 3.1) $\leq C\left(\eta,\left\|\rho_{0}(t)\right\|_{C^{0}}, \max _{i=1, \ldots, N}\left\|D \Phi_{i}\right\|_{C^{0}}\right) \sum_{j=1}^{d}\left(\left\|W_{\mu^{\prime}}^{j}\right\|_{L^{1}}+\left\|W_{\mu^{\prime \prime}}^{j}\right\|_{L^{1}}\right)$

$$
\text { (by }(51)) \leq C\left(\eta,\left\|\rho_{0}(t)\right\|_{C^{0}}, \max _{i=1, \ldots, N}\left\|D \Phi_{i}\right\|_{C^{0}}\right)\left(\frac{1}{\left(\mu^{\prime}\right)^{d-1}}+\frac{1}{\left(\mu^{\prime \prime}\right)^{d-1}}\right) \text {. }
$$

Lemma 6.7 For every $t \in[0,1]$,

$$
\left\|R^{\mathrm{corr}}(t)\right\|_{L^{1}\left(\mathbb{T}^{d}\right)} \leq C\left(M, \eta,\left\|R_{0}\right\|_{C^{1}}, \max _{i=1, \ldots, N}\left\|D \Phi_{i}\right\|_{C^{0}}\right)\left(\frac{1}{\lambda^{\prime}}+\frac{1}{\lambda^{\prime \prime}}\right) .
$$

Proof. We use Lemma 5.4 and Lemma 5.5:

$$
\begin{aligned}
\left\|\vartheta_{c}(t) w(t)\right\|_{L^{1}} & =\left|\vartheta_{c}(t)\right|\|w(t)\|_{L^{1}} \\
& \leq\left|\vartheta_{c}(t)\right|\|w(t)\|_{C^{0}} \\
& \leq C\left(M, \eta,\left\|R_{0}\right\|_{C^{1}}, \max _{i=1, \ldots, N}\left\|D \Phi_{i}\right\|_{C^{0}}\right)\left(\frac{1}{\lambda^{\prime}}+\frac{1}{\lambda^{\prime \prime}}\right) .
\end{aligned}
$$

\section{Proof of Proposition 4.1}

In this section we conclude the proof of Proposition 4.1, and thus also the proof of Theorem 1.2 and, consequently, the proof of Theorem 1.1. We first prove that if $R_{0}(t)=0$ at some time $t \in[0,1]$, then $R_{1}(t)=0$. Observe that if $R_{0}(t)=0$, then by Remark 5.2,

$$
\rho_{1}(t)-\rho_{0}(t)=\vartheta(t)+\vartheta_{c}(t)=0, \quad u_{1}(t)-u_{0}(t)=w(t)=0 .
$$

Moreover, by (54), $\psi \equiv 0$ on a neighborhood of $t$ and thus $\psi(t)=\psi^{\prime}(t)=0$. Therefore

$$
\begin{aligned}
& \psi(t)=0 \quad \Longrightarrow R^{\text {interaction }}(t)=R^{\text {flow }}(t)=R^{\text {quadr }}(t)=0, \\
& \psi(t)=\psi^{\prime}(t)=0 \quad \Longrightarrow \quad R^{\text {transport }}(t)=0, \\
& R_{0}(t)=0 \Longrightarrow R^{\psi}(t)=0 \\
& w(t)=w_{c}(t)=0 \quad \Longrightarrow \quad R^{\text {Nash }}(t)=R^{\text {corr }}(t)=0
\end{aligned}
$$

and thus $R_{1}(t)=0$.

We now prove estimates (39a)-(39d). First of all, in view of Lemma 5.5 and Lemma 6.2, we choose $\tau$ so small that

$$
\max _{i=1, \ldots, N}\left\|D \Phi_{i}^{-1}\right\|_{C^{0}\left(\operatorname{supp} \zeta_{i} \times \mathbb{T}^{d}\right)} \leq 2,
$$




$$
\left\|R_{0}\right\|_{C^{0}} \max _{i=1, \ldots, N}\left\|\mathrm{Id}-D \Phi_{i}^{-1}\right\|_{C^{0}\left(\operatorname{supp} \zeta_{i} \times \mathbb{T}^{d}\right)} \leq \frac{\delta}{4} .
$$

This is always possible since, by (53), $\Phi_{i}\left(t_{i}, x\right)=x$ and thus $D \Phi_{i}\left(t_{i}, x\right)=\operatorname{Id}$ for every $i=1, \ldots, N$. We choose also $\lambda^{\prime}, \mu^{\prime}, \lambda^{\prime \prime}, \mu^{\prime \prime}$ such that $1 \ll \lambda^{\prime} \ll \mu^{\prime} \ll \lambda^{\prime \prime} \ll \mu^{\prime \prime}$. More precisely, we set

$$
\lambda^{\prime}=\lambda, \quad \mu^{\prime}:=\lambda^{\alpha}, \quad \lambda^{\prime \prime}:=\lambda^{\beta}, \quad \mu^{\prime \prime}:=\lambda^{\gamma},
$$

for some

$$
1<\alpha<\beta<\gamma
$$

and $\lambda \gg 1$ to be fixed later.

1 Estimate (39a). If $R_{0}(t)=0$, we have already seen that $\rho_{1}(t)=\rho_{0}(t)$. We can thus assume $R_{0}(t) \neq 0$. We have

$$
\left\|\rho_{1}(t)-\rho_{0}(t)\right\|_{L^{1}} \leq\left\|\vartheta_{0}(t)\right\|_{L^{1}}+\left|\vartheta_{c}(t)\right|
$$

(by Lemmas 5.3 and 5.4)

$$
\begin{aligned}
& \leq \frac{M \eta}{2}\left\|R_{0}(t)\right\|_{L^{1}}+C\left(M, \eta,\left\|R_{0}\right\|_{C^{1}}, \max _{i=1, \ldots, N}\left\|D \Phi_{i}\right\|_{C^{0}}\right)\left(\frac{1}{\lambda^{\prime}}+\frac{1}{\lambda^{\prime \prime}}\right) \\
& \leq \frac{M \eta}{2}\left\|R_{0}(t)\right\|_{L^{1}}+C\left(M, \eta,\left\|R_{0}\right\|_{C^{1}}, \max _{i=1, \ldots, N}\left\|D \Phi_{i}\right\|_{C^{0}}\right)\left(\frac{1}{\lambda}+\frac{1}{\lambda^{\beta}}\right) \\
& \leq M \eta\left\|R_{0}(t)\right\|_{L^{1}},
\end{aligned}
$$

if the constant $\lambda$ is chosen large enough.

2 Estimate (39b). We have

$$
\begin{aligned}
\left\|u_{1}(t)-u_{0}(t)\right\|_{C^{0}} & \leq\|w(t)\|_{C^{0}} \\
(\text { by Lemma } 5.5) & \leq \frac{M}{2 \eta} \max _{i=1, \ldots, N}\left\|\left(D \Phi_{i}\right)^{-1}\right\|_{C^{0}\left(\operatorname{supp} \zeta_{i} \times \mathbb{T}^{d}\right)} \\
(\text { by }(71 \mathrm{a})) & \leq \frac{M}{\eta} .
\end{aligned}
$$

3 Estimate (39c). We have

$$
\left\|u_{1}(t)-u_{0}(t)\right\|_{W^{1, p}} \leq\|w(t)\|_{W^{1, p}}
$$

(by Lemma 5.6)

$$
\begin{aligned}
& \leq C\left(M, \eta, \max _{i=1, \ldots, N}\left\|D \Phi_{i}\right\|_{C^{1}}\right)\left(\lambda^{\prime}\left(\mu^{\prime}\right)^{1-(d-1) / p}+\lambda^{\prime \prime}\left(\mu^{\prime \prime}\right)^{1-(d-1) / p}\right) \\
& \leq C\left(M, \eta, \max _{i=1, \ldots, N}\left\|D \Phi_{i}\right\|_{C^{1}}\right)\left(\lambda^{1+\alpha(1-(d-1) / p)}+\lambda^{\beta+\gamma(1-(d-1) / p)}\right) \\
& \leq \delta
\end{aligned}
$$

if $\alpha, \beta, \gamma$ are chosen so that

$$
\begin{aligned}
& 1+\alpha\left(1-\frac{d-1}{p}\right)<0 \\
& \beta+\gamma\left(1-\frac{d-1}{p}\right)<0
\end{aligned}
$$

and $\lambda$ is large enough. 
4 Estimate (39d). Recall the definition of $R_{1}$ in (62). Using Lemmas 6.1, 6.2, 6.3, 6.4, 6.5, 6.6, 6.7 and (71b), we get

$$
\begin{aligned}
\left\|R_{1}(t)\right\|_{L^{1}} \leq & \left\|R^{\text {interaction }}(t)\right\|_{L^{1}}+\left\|R^{\text {flow }}(t)\right\|_{L^{1}}+\left\|R^{\psi}(t)\right\|_{L^{1}}+\left\|R^{\text {quadr }}(t)\right\|_{L^{1}} \\
& +\left\|R^{\text {transport }}(t)\right\|_{L^{1}}+\left\|R^{\text {Nash }}(t)\right\|_{L^{1}}+\left\|R^{\text {corr }}(t)\right\|_{L^{1}} \\
\leq & \frac{\delta}{2}+C\left(M, \eta, \delta, \tau,\left\|\rho_{0}\right\|_{C^{0}},\left\|u_{0}\right\|_{C^{1}},\left\|R_{0}\right\|_{C^{2},} \max _{i=1, \ldots, N}\left\|D \Phi_{i}\right\|_{C^{1}}\right) . \\
& \cdot\left[\frac{1}{\lambda^{\prime}}+\frac{1}{\lambda^{\prime \prime}}+\frac{1}{\left(\mu^{\prime}\right)^{d-1}}+\frac{1}{\left(\mu^{\prime \prime}\right)^{d-1}}+\frac{\lambda^{\prime} \mu^{\prime}}{\lambda^{\prime \prime}}+\frac{\lambda^{\prime}\left(\mu^{\prime}\right)^{d}}{\lambda^{\prime \prime}\left(\mu^{\prime \prime}\right)^{d-1}}\right] \\
\leq & \frac{\delta}{2}+C\left(M, \eta, \delta, \tau,\left\|\rho_{0}\right\|_{C^{0}},\left\|u_{0}\right\|_{C^{1}},\left\|R_{0}\right\|_{C^{2},} \max _{i=1, \ldots, N}\left\|D \Phi_{i}\right\|_{C^{1}}\right) . \\
& \cdot\left[\frac{1}{\lambda}+\frac{1}{\lambda^{\beta}}+\frac{1}{\lambda^{\alpha(d-1)}}+\frac{1}{\lambda^{\gamma(d-1)}}+\frac{\lambda^{1+\alpha}}{\lambda^{\beta}}+\frac{\lambda^{1+\alpha d}}{\lambda^{\beta+\gamma(d-1)}}\right] \\
\leq & \frac{\delta}{2}+\frac{\delta}{2} \leq \delta,
\end{aligned}
$$

if

$$
\begin{aligned}
1+\alpha-\beta & <0, \\
1+\alpha d-\beta-\gamma(d-1) & <0,
\end{aligned}
$$

and $\lambda$ is large enough.

We still have to choose $\alpha, \beta, \gamma$ so that (72), (73) are satisfied. This can be easily done as follows, recalling that $p<d-1$. First we fix $\alpha>1$ so that

$$
\alpha>\frac{1}{\frac{d-1}{p}-1},
$$

so that (72a) is satisfied. Then we choose $\beta$ so that

$$
\beta>1+\alpha,
$$

so that (73a) is satisfied. Finally we choose $\gamma>1$ so that

$$
\gamma>\frac{\beta}{\frac{d-1}{p}-1} \text { and } \gamma>\frac{1+\alpha d-\beta}{d-1},
$$

so that (72b) and (73b) are satisfied. This concludes the proof of Proposition 4.1 and thus also the proof of Theorem 1.2 and, consequently, the proof of Theorem 1.1.

Acknowledgements This research was supported by the ERC Grant Agreement No. 724298.

Open Access This article is distributed under the terms of the Creative Commons Attribution 4.0 International License (http://creativecommons.org/licenses/by/4.0/), which permits unrestricted use, distribution, and reproduction in any medium, provided you give appropriate credit to the original author(s) and the source, provide a link to the Creative Commons license, and indicate if changes were made.

\section{References}

1. Ambrosio, L.: Transport equation and Cauchy problem for BV vector fields. Invent. Math. 158(2), 227260 (2004) 
2. Ambrosio, L.: Well posedness of ODE's and continuity equations with nonsmooth vector fields, and applications. Rev. Mat. Complut. 30(3), 427-450 (2017)

3. Bianchini, S., Bonicatto, P.: A uniqueness result for the decomposition of vector fields in Rd. SISSA (2017)

4. Buckmaster, T., De Lellis, C., Isett, P., Székelyhidi Jr., L.: Anomalous dissipation for 1/5-Hölder Euler flows. Ann. Math. (2) 182(1), 127-172 (2015)

5. Buckmaster, T., De Lellis, C., Székelyhidi Jr., L., Vicol, V.: Onsager's conjecture for admissible weak solutions. Commun. Pure Appl. Math. 72, 229-274 (2019)

6. Buckmaster, T., Vicol, V.: Nonuniqueness of weak solutions to the Navier-Stokes equation. Ann. Math. (2) 189(1), 101-144 (2019)

7. Caravenna, L., Crippa, G.: Uniqueness and Lagrangianity for solutions with lack of integrability of the continuity equation. C. R. Math. Acad. Sci. Paris 354(12), 1168-1173 (2016)

8. Daneri, S., Székelyhidi Jr., L.: Non-uniqueness and h-principle for Hölder-continuous weak solutions of the Euler equations. Arch. Ration. Mech. Anal. 224(2), 471-514 (2017)

9. De Lellis, C., Székelyhidi Jr., L.: On admissibility criteria for weak solutions of the Euler equations. Arch. Ration. Mech. Anal. 195(1), 225-260 (2008)

10. Depauw, N.: Non unicité des solutions bornées pour un champ de vecteurs BV en dehors d'un hyperplan. C. R. Math. Acad. Sci. Paris 337(4), 249-252 (2003)

11. DiPerna, R.J., Lions, P.-L.: Ordinary differential equations, transport theory and Sobolev spaces. Invent. Math. 98(3), 511-547 (1989)

12. Evans, L.C.: Partial Differential Equations. American Mathematical Society, Providence (2010)

13. Isett, P.: A proof of Onsager's conjecture. Ann. Math. (2) 188(3), 871-963 (2018)

14. Isett, P., Oh, S.-J.: On nonperiodic Euler flows with Hölder regularity. Arch. Ration. Mech. Anal. 221(2), 725-804 (2016)

15. Modena, S., Sattig, G.: Convex integration solutions to the transport equation with full dimensional concentration. arXiv (2019)

16. Modena, S., Székelyhidi, L.: Non-uniqueness for the transport equation with Sobolev vector fields. Ann. PDE 4(2), 18 (2018)

Publisher's Note Springer Nature remains neutral with regard to jurisdictional claims in published maps and institutional affiliations. 